\title{
Co-expression clustering across flower development identifies modules for diverse floral forms in Achimenes (Gesneriaceae)
}

\author{
Wade R Roberts ${ }^{\text {Corresp., } 1,2}$, Eric H Roalson ${ }^{1}$ \\ ${ }^{1}$ School of Biological Sciences, Washington State University, Pullman, Washington, United States \\ 2 Biological Sciences, University of Arkansas, Fayetteville, Arkansas, United States \\ Corresponding Author: Wade R Roberts \\ Email address: wader@uark.edu
}

Background. Genetic pathways involved with flower color and shape are thought to play an important role in the development of flowers associated with different pollination syndromes, such as those associated with bee, butterfly, or hummingbird pollination. Because pollination syndromes are complex traits that are orchestrated by multiple genes and pathways, the gene regulatory networks have not been explored. Gene co-expression networks provide a systems level approach to identify important contributors to floral diversification.

Methods. RNA-sequencing was used to assay gene expression across two stages of flower development (an early bud and an intermediate stage) in 10 species of Achimenes (Gesneriaceae). Two stage-specific co-expression networks were created from 9503 orthologs and analyzed to identify module hubs and the network periphery. Module association with bee, butterfly, and hummingbird pollination syndromes was tested using phylogenetic mixed models. The relationship between network connectivity and evolutionary rates $\left(d_{N} / d_{s}\right)$ was tested using linear models.

Results. Networks contained 65 and 62 modules that were largely preserved between developmental stages and contained few stage-specific modules. Over a third of the modules in both networks were associated with flower color, shape, and pollination syndrome. Within these modules, several hub nodes were identified that related to the production of anthocyanin and carotenoid pigments and the development of flower shape. Evolutionary rates were decreased in highly connected genes and elevated in peripheral genes.

Discussion. This study aids in the understanding of the genetic architecture and network properties underlying the development of floral form and provides valuable candidate modules and genes for future studies. 
2 Co-expression clustering across flower development

3 identifies modules for diverse floral forms in

4 Achimenes (Gesneriaceae)

Wade R Roberts ${ }^{1,2}$, Eric H Roalson ${ }^{1}$

7

8

${ }^{1}$ School of Biological Sciences, Washington State University, Pullman, WA, United States

${ }^{2}$ Current address: Biological Sciences, University of Arkansas, Fayetteville, AR, United States 10

Corresponding Author:

12 Wade Roberts ${ }^{1}$

Biological Sciences, University of Arkansas, SCEN 601, Fayetteville, AR, 72701, United States Email address: wader@uark.edu

\section{Abstract}

Background. Genetic pathways involved with flower color and shape are thought to play an important role in the development of flowers associated with different pollination syndromes, such as those associated with bee, butterfly, or hummingbird pollination. Because pollination syndromes are complex traits that are orchestrated by multiple genes and pathways, the gene networks have not been explored. Gene co-expression networks provide a systems level approach to identify important contributors to floral diversification.

Methods. RNA-sequencing was used to assay gene expression across two stages of flower development (an early bud and an intermediate stage) in 10 species of Achimenes (Gesneriaceae). Two stage-specific co-expression networks were created from 9503 orthologs and analyzed to identify module hubs and the network periphery. Module association with bee, butterfly, and hummingbird pollination syndromes was tested using phylogenetic mixed models. The relationship between network connectivity and evolutionary rates $\left(d_{\mathrm{N}} / d_{\mathrm{S}}\right)$ was tested using linear models.

Results. Networks contained 65 and 62 modules that were largely preserved between developmental stages and contained few stage-specific modules. Over a third of the modules in both networks were associated with flower color, shape, and pollination syndrome. Within these modules, several hub nodes were identified that related to the production of anthocyanin and carotenoid pigments and the development of flower shape. Evolutionary rates were decreased in highly connected genes and elevated in peripheral genes.

Discussion. This study aids in the understanding of the genetic architecture and network properties underlying the development of floral form and provides valuable candidate modules and genes for future studies. 
39

40

41

42

43

44

45

46

47

48

49

50

51

52

53

54

55

56

57

58

59

60

61

62

63

64

65

66

67

68

69

70

71

72

73

74

75

76

77

78

\section{Introduction}

Flowers display a diverse range of colors, shapes, and sizes, and understanding the ecological and genetic factors contributing to their diversity across angiosperms has long been a major goal in biology. Often this diversity has been attributed to pollinator-mediated selection (Stebbins 1970; O’Meara et al. 2016; Gervasi and Schiestle 2017) and the relationship between a plant and its mode of pollination is considered to be one of the key innovations contributing to angiosperm diversification (Faegri and van der Pijl 1979; Fenster et al. 2004; van der Niet and Johnson 2012; Barrett 2013; Sauquet and Magallón 2018). Most plants evolved to rely on biotic or abiotic means in order to move pollen and ensure reproductive success (Faegri and van der Pij1 1979; Fenster et al. 2004). These biotic pollinators are often attracted to flowers that contain specific traits, such as red flowers that provide high nectar rewards for bird visitors (Cronk and Ojeda 2008) or the ultraviolet markings on some flowers that attract bee visitors (Papiorek et al 2016). A wide range of floral traits are thought to contribute to successful pollination, such as color (Sletvold et al. 2016), odor (Piechowski et al. 2010), nectar composition (Amorim et al. 2013), and time of flowering (Cortés-Flores et al. 2017). The genetic basis for these and other traits and their role in floral and pollination syndrome divergence has been examined extensively in model systems, particularly in Mimulus (Bradshaw et al. 1998; Yuan et al. 2016) and Petunia (Hoballah et al. 2007; Hermann et al. 2015), and more recently in many non-model systems (Wessinger et al. 2014; Alexandre et al. 2015).

Among the most widely used large-scale experimental approaches to investigate genome function are transcriptome analyses (Pickrell et al. 2010; Raherison et al. 2015), particularly in non-model organisms where no reference genome or functional genomics data exists. Changes in expression often result from the combinatorial action of genetic regulatory pathways orchestrating development and responses to environmental stimuli. Therefore, the transcriptome can be viewed as a link between the genotype and phenotype and may be acted upon through selection (Romero et al. 2012; Prasad et al. 2013). As changes in gene expression may underlie many of the phenotypic changes between species (Brawand et al. 2011; Romero et al. 2012; Uebbing et al. 2016), studying the transcriptome may shed light on important pathways and targets of selection. Phenotypic changes can frequently arise through functional changes in conserved developmental pathways among closely related species. For example, changes in enzyme function and transcriptional regulation of the anthocyanin pathway has been implicated frequently across angiosperms in the evolution of red flowers (Des Marais and Rausher 2010; Smith and Rausher 2011). It is now recognized that most genes act as members of biological pathways or of co-regulated modules (Hollender et al. 2014; Ma et al. 2018).

Here, we undertake a comparative study of gene expression during flower development in the genus Achimenes. This group is a member of the diverse African violet family (Gesneriaceae) and distributed throughout Mexico and Central America. Achimenes is a young lineage (c. 7-12 Mya; Roalson and Roberts 2016) known for its floral diversity (Figure 1), a

Peer) reviewing PDF | (2019:10:42302:1:1:NEW 10 Feb 2020) 
79 feature thought to be associated with speciation (Ramírez Roa 1987; Roalson et al. 2003). Four

80 pollination syndromes are found in Achimenes, including melittophily (bees), psychophily

81 (butterflies), euglossophily (female euglossine bees), and ornithophily (hummingbirds) (Figure

82 2). These syndromes have traditionally been defined on the basis of flower color and flower

83 shape (Figure 2; Ramírez Roa 1987) and recently through pollinator observations (Martén-

84 Rodríguez et al. 2015; Ramírez-Aguirre et al. 2019). Currently there have been pollinator

85 observations made for four Achimenes species: A. antirrhina (hummingbirds, Amazilia

86 beryllina), A. flava (bees, Anthophoridae), A. obscura (bees, Trigona fulviventris), and A. patens

87 (unidentified butterflies) (Martén-Rodriguez et al. 2015; Ramírez-Aguirre et al. 2019). Based on

88 these observations and the floral traits of these species (Figure 2), we can hypothesize the likely

89 pollinators for the other Achimenes species (Figure 2; Table 1). Repeated origins and transitions

90 between these pollination syndromes have been hypothesized in Achimenes (Roalson et al.

91 2003), making this small lineage an attractive system to understand the genetic and ecological

92 factors underlying floral diversification.

93 In this study, we aimed to characterize and compare patterns of gene co-expression

94 during flower development across 10 Achimenes species. So far, few studies have investigated

95 the genetic basis of floral development in the Gesneriaceae family (Alexandre et al. 2015;

96 Roberts and Roalson 2017). Given that bee, butterfly, and hummingbird pollination syndromes

97 evolved in parallel across Achimenes, we hypothesized that: (1) distinct sets of co-expressed

98 genes would correlate to each syndrome during flower development, and (2) genes in pathways

99 involved with flower color and shape may be central in each network during flower

100 development. Using a comparative transcriptome approach, we clustered a shared set of 9503

101 orthologs into two different co-expression networks, each corresponding to two stages of flower

102 development: an early bud stage and an intermediate stage prior to anthesis. This strategy

103 allowed us to compare gene co-expression patterns temporally. We compared these networks and

104 tested whether gene co-expression clusters correlated with different floral traits (pollination

105 syndrome, flower color, flower shape, corolla spurs). We also tested whether network properties,

106 such as connectivity and expression, influence evolutionary constraints. Our results allowed us to

107 quantify the extent of shared gene co-expression among closely related species and to identify

108 pathways and genes that may be associated with the repeated evolution of flower types

109 underlying pollinator specificity.

110

111

112

113

114

115

116

117

\section{Materials \& Methods}

\section{Plant materials}

Ten of the 26 currently recognized species in Achimenes were sampled: five from Clade 1 and five from Clade 2 (Figure 2). These species were chosen based on their hypothesized close relationships and their diversity in floral form (Figure 1; Roalson et al. 2003). On the basis of previous molecular studies (Roalson and Roberts 2016), two species were chosen as outgroups to represent related lineages: Eucodonia verticillata and Gesneria cuneifolia (Figures 1-2). All 
119

120

121

122

123

124

125

126

127

128

129

130

131

132

133

134

135

136

137

138

139

140

141

142

143

144

145

146

147

148

149

150

151

152

153

154

155

156

157

158

selected species were grown in standard greenhouse conditions at Washington State University, under 16 -hour days, $24-27^{\circ} \mathrm{C}$, and $80-85 \%$ humidity.

Whole flower buds from two stages of flower development were sampled for each species: an immature bud stage (Bud stage; Figure 1) and an intermediate stage before anthesis (D stage; Figure 1). Our definitions of the floral developmental stages in Achimenes were adapted from the stages determined for Antirrhinum majus, which uses a morphological and temporal framework (Vincent and Enrico 2004). The Bud stage represents the smallest reproductive bud distinguishable from vegetative buds, having lengths between 1-2 mm (Figure 1). The appearance is marked by the emergence of the floral primordia, no accumulation of anthocyanin pigments, and no elongation or growth of corolla tissue cells. The D stage represents a halfway point between the Bud and a pre-anthesis flower, where the length is about half the length of a fully developed flower (Figure 1). Its appearance is marked by the accumulation of anthocyanins in the corolla tissue, the cells have elongated in the corolla tube, and trichomes have emerged. Corolla spurs are found only in A. grandiflora and A. patens, and the spur begins development during the $\mathrm{D}$ stage as an outward growth of the corolla in the opposite direction from the tube opening (Figure 1).

Each stage was sampled with two or three biological replicates, contributing four or six samples for each species. The bud stage was chosen to represent a baseline level of gene expression when floral phenotypes are largely similar between species that could then be compared to the intermediate stage where floral phenotypes diverge and many important pathways involved in flower development and pigmentation show increased expression levels (Roberts and Roalson 2017). All tissues were immediately frozen into liquid nitrogen and stored at $-80^{\circ} \mathrm{C}$.

\section{RNA extraction and RNA-seq library construction}

Total RNA was extracted from frozen tissue using the RNEasy Plant Kit (Qiagen) and ribosomal depleted RNA samples were prepared using the RiboMinus Plant Kit (Thermo Fisher Scientific). Stranded libraries were prepared using the NEBNext Ultra Directional RNA Library Kit (New England Biolabs), barcoded, and pooled based on nanomolar concentrations. Library quality and quantity were assessed using the Qubit (dsDNA HS assay; Thermo Fisher Scientific) and BioAnalyzer 2100 (Agilent). The library pool was sequenced across 4 lanes of an Illumina HiSeq2500 for paired-end $101 \mathrm{bp}$ reads at the Genomics Core Lab at Washington State University, Spokane. All new sequence data were deposited in the NCBI Sequence Read Archive (BioProject: PRJNA435759).

Single replicate libraries for four of the species in the current study (A. cettoana, $A$. erecta, $A$. misera, and $A$. patens) were previously sequenced for the same two timepoints (Bud and D stages) (Roberts and Roalson 2017). These samples were combined with the newly generated data to assembly de novo transcriptomes and perform co-expression clustering. This data was deposited in the NCBI Sequence Read Archive (BioProject: PRJNA340450). 
159

160

161

162

163

164

165

166

167

168

169

170

171

172

173

174

175

176

177

178

179

180

181

182

183

184

185

186

187

188

189

190

191

192

193

194

195

196

197

198

\section{Transcriptome assembly}

Per base quality, adapter contamination, and per base sequence content of raw reads was assessed with FastQC tools (http://bioinformatics.babraham.ac.uk/projects/fastqc). One library (AT6) returned poor yields and low-quality reads and was excluded from further analyses. Trimmomatic v.0.36 (Bolger et al. 2014) was then used to remove contaminating adapter sequences and dynamically trim low-quality bases (ILLUMINACLIP:TruSeq3-PE-2.fa:2:30:10 HEADCROP:13 LEADING:3 TRAILING:3 SLIDINGWINDOW:4:15 MINLEN:50). Trimmed reads derived from an individual species were combined and de novo assembled into contigs using Trinity (--SS_lib_type RF; Grabherr et al. 2011; Haas et al. 2013), generating a reference transcriptome for each species. Sequence redundancy was reduced with CDHIT-EST (-c 0.99 -n 5; Li and Godzik 2006). Open reading frames were then predicted for each transcriptome assembly with TransDecoder (Haas et al. 2013) using a BLASTp search against the SwissProt database (www.uniprot.org) to maximize sensitivity. Finally, transcriptome assembly completeness was assessed using BUSCO (Simão et al. 2015) against a set of single-copy plant orthologs (embryophyte_odb10 dataset).

\section{Orthogroup inference}

To help facilitate orthogroup inference, transcriptome assemblies from flowers in Sinningia eumorpha and S. magnifica were downloaded and combined with our assemblies (https://doi.org/10.5061/dryad.4r5p1; Serrano-Serrano et al. 2017a). Sinningia is closely related to our focal lineage in the Neotropical Gesneriaceae (Roalson and Roberts 2016). To identify orthogroup sequences across all 14 species, we used a modified version of the approach described in Yang and Smith (2014). All peptide sequences were clustered using an all-by-all BLASTp search performed using DIAMOND (--evalue 1e-6; Buchfink et al. 2014) and results were clustered using MCL v14-137 (-I 1.4; Enright et al. 2002). Homolog clusters were aligned using MAFFT v7.271 (--genafpair --maxiterate 1000; Katoh and Standley 2013) and alignments were trimmed using Phyutility v2.7.1 (-clean 0.1; Smith and Dunn 2008). Codon alignments were then produced for each cluster using PAL2NAL v14 (Suyama et al. 2006) and maximumlikelihood trees were constructed using FastTree v2.1.8 (Price et al. 2010) using the GTR model. Trees were then trimmed to exclude branches $>10$ times longer than its sister or longer than 0.2 substitutions per site. Final homolog group alignments were created from the trimmed trees using MAFFT and used for final homolog tree inference using RAxML v8.2.9 (Stamatakis 2014) and the GTRGAMMA model. Orthogroups were inferred from the final homolog trees using the rooted ingroup (RT) method of Yang and Smith (2014), using S. eumorpha and S. magnifica as outgroups. Homolog trees were trimmed to include at least 2 ingroup species, resulting in 83595 orthogroups.

\section{Quantifying gene expression}

Trimmed reads for each sample were aligned to the corresponding transcriptome assembly to quantify expression using default parameters in Kallisto v0.43.0 (Bray et al. 2016). 
199 The resulting counts for each contig were matched with the inferred orthogroups derived above, 200 creating two separate matrices for the Bud and D stage samples. In the resulting gene expression 201 matrices, rows corresponded to orthogroups and columns corresponded to individual samples

202 (Supplemental Data). Counts for the Bud and D stage samples were separately transformed using 203 variance-stabilizing transformation implemented in the Bioconductor package DESeq2 (Love et 204 al. 2014) and quantile normalized using the Bioconductor package preprocessCore (Bolstad 205 2018). Lastly, counts for both stages were separately corrected for confounding effects, such as 206 batch or species effects, using principal component regression with the 'sva_network' function in 207 208 209

\section{Co-expression networks and identification of modules}

The Bud and D stage RNAseq data was clustered into gene co-expression networks using the R package WGCNA (Langfelder and Horvath 2008). Prior to network construction, orthogroups were filtered to remove any with $>50 \%$ missing values or $<0.3$ expression variance. In total, 9503 orthogroups common to both Bud and D stage data were used to construct separate networks for each stage. Parameters for the Bud stage network were as follows: power $=18$, networkType $=$ "signed", corType $=$ "bicor", maxPOutliers $=0.05$, TOMType $=$ "signed", deepSplit $=2$, mergeCutHeight $=0.25$. For the $\mathrm{D}$ stage network, the following parameters were used: power $=16$, networkType $=$ "signed", corType $=$ "bicor", maxPOutliers $=0.05$, TOMType $=$ "signed", deepSplit $=2$, mergeCutHeight $=0.25$. Soft-thresholding powers for each network ("power" parameter) were chosen as the lowest value such that the scale free topology model fit $\left(R^{\wedge} 2\right)$ was $\geq 0.9$ (Supplemental Data). Signed networks consider positively correlated nodes connected, while unsigned networks consider both positively and negatively correlated nodes connected (van Dam et al. 2018). We used signed networks here ("networkType" and "TOMType" parameters) because this type is considered more robust to biological functions with more specific expression patterns (Mason et al. 2009; Song et al. 2012). Unsigned networks can capture only strong correlations and many negative regulatory relationships in biological systems are weak or moderate (Ritchie et al. 2009). Biweight midcorrelation ("corType" parameter) was used as this method is often more robust than Pearson correlation and more powerful than Spearman correlation (Song et al. 2012). All other WGCNA parameters were kept at their default values.

Module eigengenes and orthogroup connectivity were calculated in each network separately using the 'moduleEigengenes' and 'intramodularConnectivity' functions in WGCNA, respectively. The module eigengene is defined as the first principal component of a module and represents the gene expression profile of a sample within a module (Langfelder and Horvath 2008). Connectivity refers to the sum of connection strengths a node has to other network nodes. Module hub genes (defined as the most highly connected nodes within a module) were identified in both networks based on module membership (kME) scores $>0.9$, calculated using the 'signedKME' function of WGCNA. kME is defined as the correlation between the expression levels of a gene and the module eigengene (Horvath and Dong 2008). Additionally, 
239

240

241

242

243

244

245

246

247

248

249

250

251

252

253

254

255

256

257

258

259

260

261

262

263

264

265

266

267

268

269

270

271

272

273

274

275

276

277

278

using connectivity measures, we defined peripheral genes in each network as the lowest $10 \%$ of connected nodes.

We tested whether Bud stage modules were preserved in the D stage and vice versa using module preservation statistics in WGCNA (Langfelder et al. 2011). Median Rank and Zsummary statistics were computed using the 'modulePreservation' function of WGCNA, using 200 permutations (Langfelder et al. 2011). Median rank statistics were compared to the "gold" module, which consists of 1000 randomly selected orthogroups. Modules with a lower median rank exhibit stronger preservation than modules with a higher median rank. Zsummary scores $>$ 10 indicate strong evidence of preservation and scores $<2$ indicate no evidence of preservation.

\section{Phylogeny}

We previously inferred a phylogeny for the 12 sampled species using 1306 single-copy orthologs identified from the same transcriptome dataset used here (Roberts and Roalson 2018). For comparative analyses of module-trait relationships, we randomly sampled 50 single-copy ortholog gene trees and rescaled branch lengths to be proportional to time (ultrametric) using the 'chronos' function in the R package ape (Paradis et al. 2004). Bootstrap support was 100 for nearly every branch in the Roberts and Roalson (2018) phylogeny, therefore we chose to use randomly sampled ortholog trees to account for phylogenetic uncertainty.

\section{Module and trait relationship}

We correlated external traits (e.g., red flower color) with the module eigengenes using a phylogenetic Markov Chain Monte Carlo method, implemented in the R packages MCMCglmm (Hadfield 2010) and mulTree (Guillerme and Healy 2014). These analyses were performed individually for each module in both the Bud and D stage networks. Three floral traits and pollination syndrome were coded: primary flower color (purple, red, white, yellow), flower shape (funnelform, salverform, tubular), corolla spur (absent, present), and syndrome (bee, butterfly, hummingbird). We fit a multivariate mixed model with a non-informative prior, where the shape and scale parameters equal 0.001, and residual variance was fixed. Analyses were performed over the 50 randomly selected ortholog trees to account for phylogenetic uncertainty. A phylogenetic model was used to account for any interspecific non-dependency in the dataset by using a random effects structure that incorporated a phylogenetic tree model. Floral traits were set as categorical response variables and the module eigengenes were set as the predictor variable. For each tree, two MCMC chains were run for $250 \mathrm{k}$ generations and discarding the first $50 \mathrm{k}$ as burn-in. This process was run individually over each module in both the Bud and D stages. We checked for convergence between model chains using the Gelman-Rubin statistic (Gelman and Rubin 1992). Potential scale reduction values were all less than 1.1 and effective sample sizes for all fixed effects were greater than 400 . We considered fixed effects to be statistically significant when the probabilities in the $95 \%$ credible region did not include zero (Supplemental Figures 3-6). 
279

280

281

282

283

284

285

286

287

288

289

290

291

292

293

294

295

296

297

298

299

300

301

302

303

304

305

306

307

308

309

310

311

312

313

314

315

316

317

318

\section{Evolutionary rate analysis}

Codon alignments of the filtered orthogroups $(n=9503)$ were produced using PAL2NAL v14 (Suyama et al. 2006). Subsequent alignments and corresponding gene trees were used as input to the codeml package in PAML v4.9 (Yang 2007) to estimate $d_{\mathrm{N}} / d_{\mathrm{S}}($ omega, $\omega)$. $d_{\mathrm{N}} / d_{\mathrm{S}}$ for each orthogroup was estimated using a one-ratio model (model $=0, \mathrm{NSsites}=0$ ), providing a single estimate for each orthogroup to match other single orthogroup metrics, such as connectivity. If $d_{\mathrm{N}} / d_{\mathrm{S}}$ values were exceptionally large $(=999)$ because of zero synonymous differences, those values were removed from the dataset.

First, we performed linear regression (LM) to test the effect of orthogroup connectivity, average expression levels, and their interactions (explanatory variables) on the estimated $d_{\mathrm{N}} / d_{\mathrm{S}}$ values (main effect), $\mathrm{LM}=d_{\mathrm{N}} / d_{\mathrm{S}} \sim$ connectivity * expression. This was performed using the $1 \mathrm{~m}$ function in $\mathrm{R}$ with 10000 bootstrap pseudoreplicates. Second, we ran two-sample t-tests with 10000 permutations to test whether hub nodes and periphery nodes in each network had lower (for hubs) or higher (for periphery) $d_{\mathrm{N}} / d_{\mathrm{S}}$ values than all background nodes. Third, we fit a LM with 10000 bootstrap pseudoreplicates to test whether modules associated with a pollination syndrome (bee, butterfly, or hummingbird; see Results below) had increased $d_{\mathrm{N}} / d_{\mathrm{S}}$ values, LM= $d_{\mathrm{N}} / d_{\mathrm{S}} \sim$ syndrome. For all LM and permutation analyses, $d_{\mathrm{N}} / d_{\mathrm{S}}$, connectivity, and expression values were log transformed to reach a normal distribution before being processed. Analyses were performed separately for each network using the separately estimated connectivity and expression levels.

\section{Functional annotation and GO enrichment analysis}

Filtered orthogroups ( $\mathrm{n}=9503)$ were annotated based on a search against all green plant proteins in the SwissProt database (release 2018_07; The UniProt Consortium 2017) using Diamond BLASTp (--evalue 1e-6). Results were used to assign annotations and gene ontology (GO) terms. GO enrichment analyses were performed using the R package TopGO with the default "weight01" algorithm with the recommended cutoff of $\mathrm{p}<0.05$ (Alexa et al. 2006). Enriched GO term redundancy was removed and summarized using the semantic similarity measures implemented in the REVIGO web server (Supek et al. 2011).

\section{Data and code availability}

Raw sequencing reads are available from the NCBI Sequence Read Archive under BioProjects: PRJNA435759 and PRJNA340450. Data and code for all analyses can found from https://drive.google.com/open?id=1pvzw1t1pObY_kAEYmxQ484PCTuEWrsrb.

\section{Results}

\section{Transcriptome assembly}

Twelve transcriptomes were sequenced (72 libraries) and assembled in 12 species after sampling across two floral developmental stages (Supplemental Table 1). The transcriptomes had 
319

320

321

322

323

324

325

326

327

328

329

330

331

332

333

334

335

336

337

338

339

340

341

342

343

344

345

346

347

348

349

350

351

352

353

354

355

356

357

358

an average assembly length of $244.9 \mathrm{Mb}( \pm 33.9 \mathrm{Mb})$, an average of 217,510 $( \pm 47 \mathrm{k})$ transcripts, and an average N50 length of $1988 \mathrm{bp}$ ( $\pm 306 \mathrm{bp}$ ). Between 22,942 and 33,501 putative genes were detected with 2.34 ( \pm 0.35$)$ putative isoforms. BUSCO identified an average of $87 \%$ of the complete, conserved single-copy plant orthologs (Supplemental Table 1). Our focus in our comparative study was on the presence of conserved orthologs, and not on the presence of taxonomically restricted genes, which are not likely preserved across species.

\section{Constructing the co-expression network for 12 species}

We identified 83595 orthologous clusters (orthogroups), each containing at least 2 species. After removing 74092 orthogroups with too many missing values ( $>50 \%$ missing) or low expression variance ( $<0.3$ variance) and keeping common orthogroups shared between the Bud and D stages, 9503 orthogroups were used to construct two co-expression networks using the weighted gene correlation network analysis (WGCNA) approach. Modules of co-expressed orthogroups are inferred using the expression profiles of each sample regardless of species. Clustering identified 65 and 62 co-expression modules in the Bud and D stage networks, respectively (Figure 3; Supplemental Table 2). Module size in the Bud stage ranged from 22 (module ME63) to 592 (ME1) orthogroups (mean = 146) and ranged from 21 (ME61) to 704 $(\mathrm{ME} 1)$ orthogroups $($ mean $=153)$ in the D stage. Out of the 9503 orthogroups, $7845(83 \%)$ and $8735(92 \%)$ were assigned to modules in each network.

The remaining unassigned genes from each network that were not placed into a welldefined module were assigned to the ME0 module by WGCNA. Despite being unplaced, these orthogroups may still be functionally relevant within each network. Unassigned genes in the ME0 module were enriched for many processes, including transcription (Bud, $\mathrm{n}=173 ; \mathrm{D}, \mathrm{n}=$ 84 ) and organ development $(D, n=55)$, among other functions (Supplemental Tables 3-4).

We tested whether modules in the Bud stage were preserved in the D stage, and vice versa. Overall, preservation of modules between the Bud and D stage networks was high. Eight of the 65 Bud stage modules (ME46, ME62, ME44, ME57, ME42, ME41, ME55, ME40) were not strongly preserved in the D stage, while only 1 of the 62 D stage modules (ME59) was not strongly preserved in the Bud stage (Supplemental Table 5). These patterns suggest that these non-preserved modules may play unique roles within their network. Among the 8 Bud stagespecific modules were many orthogroups related to primary cell growth, meristem identity and differentiation, stamen development, and flowering (Supplemental Table 3). In the single D stage-specific module were many orthogroups related to methylation, flowering, and carotenoid biosynthesis (Supplemental Table 4). Despite the relatively high preservation, correspondence between module membership in the Bud and D stages was moderate (Figure 4). Orthogroup module membership tended to shift between each network. For example, most members of module ME15 in the Bud stage are transferred to modules ME3 and ME7 in the D stage (Figure 4).

We defined network hubs (the most highly connected nodes within a module) with module membership (kME) scores $>0.9$. Using this definition, all modules in each network 
359

360

361

362

363

364

365

366

367

368

369

370

371

372

373

374

375

376

377

378

379

380

381

382

383

384

385

386

387

388

389

390

391

392

393

394

395

396

397

398

contained at least one hub, with the percentage of hubs ranging from $1 \%$ to $24 \%$ of orthogroups in a module (Figures 5-6; Supplemental Table 6). Of the 632 and 691 hubs identified in the Bud and D stages, a significant portion (110) were shared between networks (Fisher's Exact Test, odds ratio $=3.02, \mathrm{P}<0.001)$. Functional enrichment of hubs in the Bud stage revealed that many were involved in the regulation of development and metabolic processes $(n=40)$, transport and protein localization $(n=39)$, biosynthetic processes $(n=19)$, and DNA methylation $(n=7)$, consistent with the notion that hubs usually play important roles in integrating other genes during network evolution (Ravasz et al. 2002) (Table 2). In the D stage, hubs were enriched for functions related to translation $(n=33)$, transport $(n=9)$, ethylene and abscisic acid signaling pathways $(n=17)$, carotenoid and anthocyanin biosynthetic processes $(n=11)$, and the regulation of gene expression $(n=20)$, among others (Table 2$)$.

Nodes in the periphery of each network were loosely defined by considering them to be the lowest $10 \%$ connected (connectivity ${ }_{\text {Bud }}<2.824$, connectivity ${ }_{D}<4.651$ ). These definitions identified 951 periphery nodes (Supplemental Table 7), of which 301 were peripheral in both networks (Fisher's Exact Test, odds ratio $=5.67, \mathrm{P}<0.001$ ). These periphery nodes in the Bud stage were enriched for functions related to reproductive structure development $(n=75)$, transport and localization $(\mathrm{n}=107)$, and signaling $(\mathrm{n}=389)$, among others (Table 3$)$. In the D stage, the periphery was enriched for functions related to the regulation of flower development (n $=64)$, the regulation of flowering time $(n=18)$, the regulation of flavonoid biosynthesis $(n=7)$, and cell fate specification $(n=12)$, among others (Table 3). Greater than expected numbers of peripheral nodes in the D stage were related to regulation (Fisher's exact test, odds ratio $=1.191$, $\mathrm{p}=0.02752$ ) and transcription (Fisher's exact test, odds ratio $=1.370, \mathrm{p}=0.001549$ ). In contrast, there were no differences in the expected number of peripheral nodes related to regulation (Fisher's exact test, odds ratio $=0.955, \mathrm{p}=0.7044$ ) or transcription (Fisher's exact test, odds ratio $=1.047, \mathrm{p}=0.3549$ ) in the Bud stage.

\section{Modules associated with floral traits}

We calculated module eigengenes, single values which represent the gene expression profiles of a sample within a module (Supplemental Figures 1-2). The extent of module involvement in various biological processes can be tested by correlating eigengenes with external traits, such as flower color. We tested the relationship between the module eigengenes and the four floral traits (pollination syndrome [bee, butterfly, hummingbird], flower color [purple, red, yellow, white], flower shape [funnelform, salverform, tubular], and corolla spurs [absence, presence]), while controlling for any phylogenetic bias in the dataset using a phylogenetic mixed model (Figures 5-6; Supplemental Figures 3-6). We also examined whether eigengene values (> 0.1 or $<-0.1)$ in single species contributed to a strong correlation or whether eigengene values $(>$ 0.1 or $<-0.1$ ) in multiple species contributed to a strong correlation (Figures 5-6; Supplemental Figures 3-6). We considered there to be strong evidence for trait association when a significant correlation from MCMCglmm was coupled with elevated eigengene $(>0.1)$ or decreased eigengene $(<-1.0)$ expression in multiple species that shared any of the significant traits. 
399

400

401

402

403

404

405

406

407

408

409

410

411

412

413

414

415

416

417

418

419

420

421

422

423

424

425

426

427

428

429

430

431

432

433

434

435

436

437

438

Likewise, weak evidence for trait association was considered when the MCMCglmm results were significant, but only a single species or no species sharing the significant traits had elevated $(>0.1)$ or decreased $(<-1.0)$ eigengene expression. WGCNA does not use trait information when inferring modules, so modules that correlated strongly with any floral trait were inferred without a priori knowledge.

Pollination syndrome-We included 3 species with the bee pollination syndrome $(A$. candida, A. misera, and E. verticillata), 4 species with the butterfly pollination syndrome ( $A$. cettoana, A. grandiflora, A. longiflora, and A. patens 'Major'), and 5 species with the hummingbird pollination syndrome (A. admirabilis, A. antirrhina, A. erecta, A. pedunculata, and G. cuneifolia) (Figure 2; Table 1). These pollination syndromes are traditionally defined in Achimenes and other gesneriads based on primary flower color, flower shape, and the presence of corolla spurs (Wiehler 1983; Ramírez Roa 1987; Roalson et al. 2003). Expression of 23 (35\%) and $21(34 \%)$ modules were positively associated with the pollination syndromes in the Bud and D stage networks, consisting of 2967 and 2880 orthogroups, respectively. (Figures 5-6). There were $3(4.6 \%), 7(10.8 \%)$, and $13(20 \%)$ modules associated with bee, butterfly, and hummingbird pollination syndrome in the Bud stage network, while $1(1.5 \%), 8(12.9 \%)$, and 12 (19.4\%) modules were associated with the same syndromes in the D stage. Modules were never associated with more than one syndrome. Butterfly and hummingbird syndromes were never correlated to the same modules and were often correlated in opposite directions (i.e., butterfly was positive correlated and hummingbird was negative correlated).

Among the 3 modules that were correlated to the bee pollination syndrome in the Bud stage were many orthogroups involved in hormone signaling pathways (particularly ethylene and abscisic acid, $n=14)$, cell wall and lipid biosynthesis $(n=7)$, photomorphogenesis $(n=2)$, nitrogen compound metabolism $(n=4)$, and the transport of potassium and monocarboxylic acids $(n=5)$ (Table 4$)$. In contrast, the 1 module correlated to the bee pollination syndrome in the D stage was enriched for different functions from the Bud stage, including the positive regulation of molecular functions and response to biotic stimuli $(n=7)$, phospholipid catalysis $(n$ $=2)$, xyloglucan biosynthesis $(n=2)$, mRNA stability and catalysis $(n=5)$, and chromatin modification $(n=4)$ (Table 5).

The 7 modules correlated to the butterfly pollination syndrome in the Bud stage contained orthogroups involved in RNA splicing $(n=16)$, fertilization $(n=3)$, DNA methylation $(n=2)$, cell growth and shoot formation $(n=8)$, the development of organ boundaries $(n=3)$, and stress responses $(n=83)$ (Table 4$)$. Within the 8 modules correlated with butterfly pollination in the D stage were orthogroups enriched for functions in photorespiration $(n=7)$, microtubule organization $(n=5)$, chromatin silencing $(n=3)$, red and far-red light responses $(n$ $=3)$, modified amino acid biosynthesis and metabolism $(\mathrm{n}=10)$, and mRNA transport $(\mathrm{n}=9)$ (Table 5).

Thirteen modules were correlated to the hummingbird pollination syndrome in the Bud stage and the orthogroups were enriched for numerous functions, including many developmental processes (Table 4). These modules were enriched for floral whorl development $(n=12)$, RNA 
439 and mRNA modifications $(\mathrm{n}=19)$, cell growth and division $(\mathrm{n}=54)$, organelle assembly $(\mathrm{n}=3)$, 440 transportation of carbohydrates and potassium $(\mathrm{n}=22)$, and responses to external stimuli $(\mathrm{n}=$ 441 17) (Table 4). Many of the same enrichments were seen in the 12 modules correlated to the 442 hummingbird pollination syndrome in the D stage. These modules had enrichments for floral 443 whorl development $(n=14)$, cell polarity and proliferation $(n=8)$, adaxial/abaxial specification $444(n=8)$, organ formation $(n=3)$, the regulation of growth $(n=26)$, and endosperm development $445(n=6)$. Additionally, these modules had enrichment for RNA and mRNA modification $(n=79)$, 446 transcription $(n=3)$, protein-DNA complex formation $(n=7)$, signaling $(n=25)$, and UV 447 response $(\mathrm{n}=9)$ (Table 5).

$448 \quad$ Flower color - Primary flower color is closely associated to pollination syndrome in 449 Achimenes (Ramírez Roa 1987; Roalson et al. 2003), and we identified 26 (40\%) and 17 (27\%) 450 modules associated to any flower color in the Bud and D stage networks, consisting of 2935 and

451

452

453

454

455

456

457

458

459

460

461

462

463

464

465

466

467

468

469

470

471

472

473

474

475

476

477

478 1500 orthogroups respectively (Figures 5-6). Red and yellow are associated with hummingbird pollination, purple with butterfly pollination, and white with bee pollination. In the Bud stage, there were $14(21.5 \%), 11(16.9 \%)$, and $1(1.5 \%)$ module associated with red, purple, or yellow flower color. In the D stage, no modules were associated with yellow, but $8(12.9 \%)$ and 9 $(14.5 \%)$ modules were associated with red or purple flower color. In both networks, no modules were corelated to white flower color. Similar to syndrome, purple and red flower color were never associated with the same modules and were always correlated in opposite directions.

The 14 modules associated with red flowers in the Bud stage overlapped significantly with the modules associated with the hummingbird pollination syndrome in the Bud stage and contained the same functional enrichments (Table 4; Supplemental Table 8). However, in the 8 D stage modules associated with red flowers, the orthogroups were enriched for functions involved in flower morphogenesis $(n=8)$, cell wall modification $(n=3)$, positive regulation of gene expression $(n=14)$, and histone modification $(n=7)$, among others (Supplemental Table 8).

Within the 11 modules associated with purple flowers in the Bud stage were orthogroups enriched for transcription $(n=14)$, meristem initiation $(n=4)$, DNA methylation $(n=3)$, fertilization $(n=5)$, ethylene signaling $(n=5)$, response to biotic stimuli $(n=55)$, and others (Supplemental Table 8). In contrast, the 9 modules associated with purple flowers in the D stage were enriched for orthogroups involved in anther and endosperm development $(n=9)$, translation $(n=4)$, the regulation of developmental processes $(n=9)$, lipid modification $(n=8)$, cell division $(\mathrm{n}=3)$, and hormone signaling (gibberellic acid and jasmonic acid, $\mathrm{n}=6$ ), among many other processes (Supplemental Table 8).

A single module (ME36) was associated with yellow flower color, only in the Bud stage, and contained few orthogroups involved in floral meristem determinacy $(n=1)$, floral organ identity $(n=1)$, gene silencing $(n=1)$, sulfur compound biosynthesis $(n=2)$, and vegetative phase change $(\mathrm{n}=1)$ (Supplemental Table 8$)$.

Flower shape - Flower shape is also important but not as closely associated to pollination syndromes as primary flower color in Achimenes (Ramírez Roa 1987; Roalson et al. 2003).

Flowers with bee pollination tend to have funnelform flowers, butterfly pollinated flowers all 
479 have salverform flowers, and hummingbird pollinated flowers have either salverform or tubular 480 flowers (Table 2). Overall, there were $14(22 \%)$ and 13 (21\%) modules associated with any of 481 the flower shapes in the Bud and D stages, consisting of 2940 and 2504 orthogroups, 482 respectively (Figures 5-6). Here, there were $1(1.5 \%), 8(12.3 \%)$, and $5(7.7 \%)$ modules whose 483 expression correlated to funnelform, salverform, or tubular flowers in the Bud stage (Figure 5). 484 Fewer modules were correlated in the D stage network, but expression in $1(1.6 \%), 7(12.3 \%)$, 485 and $5(8.1 \%)$ modules were associated with funnelform, salverform, and tubular shapes (Figure 486 4). No modules were positively associated with more than one flower shape.

Only two modules were associated with funnelform flowers, one in the Bud stage and the other in the D stage, and these were enriched for functions related to ethylene signaling ( $n=3)$, lipid biosynthesis $(n=3)$, chromatin modification $(n=4)$, cell wall organization $(n=2)$, and many metabolic processes (Supplemental Table 8). Within the 8 modules associated with salverform flowers in the Bud stage, there were many orthogroups involved in mRNA processing and modification $(n=50)$, ovule development $(n=7)$, chlorophyll biosynthesis $(n=4)$, and the regulation of flower development $(n=20)$ (Supplemental Table 8). In the D stage, the 7 modules associated with salverform flowers contained orthogroups involved in chromatin silencing $(\mathrm{n}=$ 7), anthocyanin biosynthesis $(n=10)$, cell cycle and cell division $(n=95)$, stamen development $(\mathrm{n}=12)$, auxin biosynthesis $(\mathrm{n}=7)$, and transport $(\mathrm{n}=17)$ (Supplemental Table 8).

Among the 5 modules associated with tubular flowers in the Bud stage were orthogroups enriched for functions in the positive regulation of transcription and organelle assembly $(n=13)$, RNA processing $(n=5)$, xylem development $(n=4)$, metabolism $(n=12)$, and many biosynthetic processes (Supplemental Table 8). In the 5 modules associated with tubular flowers in the D stage were enrichments for floral whorl development $(n=6)$, positive regulation of gene expression $(\mathrm{n}=15)$, mRNA modification $(\mathrm{n}=5)$, and signaling $(\mathrm{n}=12)$ (Supplemental Table 8).

Corolla spurs-The presence of corolla spurs is found only on butterfly pollinated species, two of which were included: A. grandiflora and A. patens (Figure 1; Table 1). There was expression in $5(7.7 \%)$ modules associated with spurs in the Bud stage network, while only 1 (1.6\%) module was associated in the D stage network (Figures 3-4). Within the 5 modules associated with corolla spurs in the Bud stage were orthogroups related to miRNA processing (n $=4)$, mRNA splicing $(n=9)$, the regulation of signal transduction pathways $(n=16)$, and pollen tube growth $(\mathrm{n}=6)$ (Supplemental Table 8$)$. In the single module associated with corolla spurs in the D stage (ME53) were very few orthogroups involved in microtubule organization $(n=2)$, stamen development $(n=1)$, the positive regulation of cell division $(n=1)$, flavonoid biosynthesis $(\mathrm{n}=1)$, and indole acetic acid metabolism $(\mathrm{n}=1)$ (Supplemental Table 8).

Non-associated-There were 13 and 9 modules not associated with any traits in the Bud and D stage networks, respectively (Figures 5-6). Three of these modules in the Bud stage were not-preserved in the D stage (modules ME46, ME44, and ME41; Figures 5-6; Supplemental Table 5). The 9 non-associated modules in the D stage were also not associated with any traits in the Bud stage (Figures 5-6) and the orthogroups had overlapping enrichments for many essential processes (Supplemental Table 8). These modules were enriched for orthogroups involved in the 
519 development of the inflorescence, stamens, and floral organs, biosynthesis of vitamins and 520 nucleotides, photosynthesis and photosynthetic electron transport, metabolism of phosphate 521 compounds, and cell surface receptor signaling (Supplemental Table 8). Numerous orthogroups 522 were also involved in the positive regulation of reproduction, the negative regulation of growth,

523 and the regulation of heterochronic development, histone methylation, and gibberellic acid 524 mediated signaling (Supplemental Table 8).

525

526

527

528

529

530

531

532

533

534

535

536

537

538

539

540

541

542

543

544

\section{Evolutionary rates correlate to network location}

We ran several analyses to explore how several network variables may affect evolutionary rates of the orthogroups within each network (as measured by $d_{\mathrm{N}} / d_{\mathrm{S}}$ ). First, we tested the effects of orthogroup connectivity, expression levels, and their interaction on the $d_{\mathrm{N}} / d_{\mathrm{S}}$ values (Supplemental Table 7). Results indicated a collective effect between connectivity and expression on $d_{\mathrm{N}} / d_{\mathrm{S}}$ in both the Bud stage $\left(\mathrm{LM}, F(3,9548)=92.58, \mathrm{p}=0, \eta^{2}=0.03\right)$ and the $\mathrm{D}$ stage networks ( $\left.\mathrm{LM}, F(3,9548)=78.36, \mathrm{p}=0, \eta^{2}=0.02\right)$. We found that orthogroup connectivity was negatively correlated with $d_{\mathrm{N}} / d_{\mathrm{S}}$ in the Bud stage network (LM, $\left.\mathrm{t}=-16.31, \mathrm{p}=0\right)$ and the $\mathrm{D}$ stage network (LM, $\mathrm{t}=-15.13, \mathrm{p}=0$ ) (Figure 7), while expression was positively correlated with $d_{\mathrm{N}} / d_{\mathrm{S}}$ in the Bud stage $(\mathrm{LM}, \mathrm{t}=2.12, \mathrm{p}=0.0344)$ and not in the D stage $(\mathrm{LM}, \mathrm{t}=1.51, \mathrm{p}=$ 0.1324) (Supplemental Figure 7). The interaction between connectivity and expression also indicated a marginal dependency of both predictors on $d_{\mathrm{N}} / d_{\mathrm{S}}$ in the Bud stage (LM, $\mathrm{t}=-2.022, \mathrm{p}$ $=0.0433)$ but not in the $\mathrm{D}$ stage network (LM, $\mathrm{t}=-1.823, \mathrm{p}=0.0683)$. These patterns have been observed in model species and may be universal to all eukaryotes.

Second, we tested whether the nodes we defined as hubs in each network had lower $d_{\mathrm{N}} / d_{\mathrm{S}}$ values, suggestive of increased evolutionary constraint (Supplemental Table 9). Hubs are considered to functionally significant and may control large portions of the network. We found that the hubs in the Bud stage network had lower $d_{\mathrm{N}} / d_{\mathrm{S}}$ than all background genes (Two-sample t-test, $t(679.31)=7.0934, \mathrm{p}=1.65 \mathrm{e}-12$, Cohen's $\mathrm{d}=0.383$; Permutation t-test. mean diff. $=$

545 $0.266, p=0.0001$ ) (Supplemental Figure 8). Similarly, hubs in the D stage network also had lower $d_{\mathrm{N}} / d_{\mathrm{S}}$ than all background genes (Two-sample t-test, $t(755.91)=4.1918, \mathrm{p}=1.55 \mathrm{e}-05$,

547 Cohen's $d=0.206$; Permutation t-test, mean diff. $=0.143, p=0.0001)($ Supplemental Figure 8).

548

549

550

551

552

553

554

555

556

557

558
Third, we tested whether the most peripheral nodes (the lowest $10 \%$ connected nodes) had higher $d_{\mathrm{N}} / d_{\mathrm{S}}$ values, which may suggest relaxed evolutionary constraint (Supplemental Table 9). These nodes are loosely connected within the network and their roles may be more apt to fluctuate during evolution and development. These peripheral genes had higher $d_{\mathrm{N}} / d_{\mathrm{S}}$ than all background genes in the Bud stage network (Two-sample t-test, $t(1487.70)=-10.272, p=0$, Cohen's $\mathrm{d}=-0.253$; Permutation t-test, mean diff. $=-0.176, \mathrm{p}=0.0001)$ and the $\mathrm{D}$ stage network (Two-sample, $t(1512.30)=-10.387, \mathrm{p}=0$, Cohen's $\mathrm{d}=-0.252$; Permutation t-test, mean diff. $=$ $0.175, \mathrm{p}=0.0001)$ (Supplemental Figure 8).

Lastly, we tested whether modules correlated with bee, butterfly, or hummingbird pollination syndromes showed increased evolutionary rates over the non-associated modules. Results indicated a very weak effect of syndrome association on $d_{\mathrm{N}} / d_{\mathrm{S}}$ in the Bud network (LM, 
559

560

561

562

563

564

565

566

567

568

569

570

571

572

573

574

575

576

577

578

579

580

581

582

583

584

585

586

587

588

589

590

591

592

593

594

595

596

597

598

$\left.F(3,9548)=5.186, \mathrm{p}=0.001406, \eta^{2}=0.0016\right)$ and the D network $(\operatorname{LM}, F(3,9548)=4.277, \mathrm{p}=$ $\left.0.005038, \eta^{2}=0.0013\right)$ (Supplemental Table 9). Only bee syndrome associated modules had marginally lower $d_{\mathrm{N}} / d_{\mathrm{S}}$ values in both networks (Bud stage, $\mathrm{LM}, \mathrm{t}=-3.58, \mathrm{p}=0.000346$; D stage, $\mathrm{LM}, \mathrm{t}=-2.508, \mathrm{p}=0.0122)$. Butterfly associated modules had marginally lower $d_{\mathrm{N}} / d_{\mathrm{S}}$ only in the D stage ( $\mathrm{LM}, \mathrm{t}=-2.548, \mathrm{p}=0.0108$ ), while hummingbird associated modules did not have any effect in either network.

\section{Discussion}

Here we report on patterns of gene co-expression across two stages of flower development in 12 species of Achimenes, Eucodonia, and Gesneria. Our comparative and phylogenetic context allowed us to reveal gene co-expression patterns across a group of closely related plant species that correlated to diverse pollination syndromes and floral traits, including flower color, flower shape, and corolla spurs. Our results suggest floral forms that are associated with bee, butterfly, and hummingbird pollinator groups are correlated with many co-expression modules. We found that nearly a third of modules in each network (23 and 21 modules in the Bud and D stages, respectively) had evidence for association with the three pollination syndromes (Figures 5-6). In our analysis, large fractions of our 9503 orthogroups set could be partitioned into modules (Figure 3), network hubs and peripheral nodes identified (Supplemental Tables 6-7), and several orthogroups were identified for their potential importance in floral phenotypic differentiation. Lastly, orthogroup position in each network (measured by connectivity) had an association with evolutionary rate $\left(d_{\mathrm{N}} / d_{\mathrm{S}}\right)$ (Figure 7$)$. These analyses provide a first overview of floral transcriptional architecture across members of the same genus that display diverse floral forms.

\section{Hub nodes contain important candidates for floral form}

To date, a large number of studies conducted in diverse angiosperm systems, have found that many genes are associated with flower development (Quattrocchio et al. 1999; Howarth et al. 2011). Despite evidence for extensive gene/genome duplications that could expand the genetic repertoire (Tang et al. 2008), many recent studies have suggested that the generation of novel genes is rare (Patterson et al. 2009; De Smet et al. 2013). Thus, the evolution and development of diverse floral forms may predominantly occur through the co-option of existing pathways (Preston et al. 2011). To our knowledge, gene co-expression network analyses have not been previously used across multiple species in a non-model lineage to understand flower development and evolution.

Hub nodes (genes) are considered to be conserved, highly connected nodes that are central to network architecture and may directly orchestrate numerous biological processes (Ravasz et al. 2002; Hu et al. 2016; Mähler et al. 2017). Our analyses identified over 600 gene nodes as hubs within each network, the majority of which were only hubs within a specific floral stage. These hub nodes tended to have lower $d_{\mathrm{N}} / d_{\mathrm{S}}$ estimates, suggesting that they not prone to rapid evolutionary change (Supplemental Table 9). Unsurprisingly, hub nodes in the Bud stage 
599 were enriched for many processes related to early flower development, including cell growth, 600 leaf formation, and photosystem assembly (Table 2). Similarly, hub nodes in the D stage were 601 enriched for genes that may be related the development of distinct floral forms, including 602 carotenoid biosynthesis, anthocyanin biosynthesis, and cell morphogenesis (Table 2). Within this 603 context of flower development, we identified a number of hubs that we consider important 604 candidates for involvement in the production of flower color and the development of floral form. 605 Many of these candidates were considered previously (Roberts and Roalson 2017) as potentially 606 important for the diversification of floral form in Achimenes. Undoubtedly, this list of hubs 607 contains many genes that may important for other biological traits that we do not consider here, 608 such as scent, nectar, and pollen production.

609 Anthocyanin biosynthesis-Anthocyanins are common floral pigments that produce red, 610 purple, and blue colors and are produced via the highly conserved flavonoid biosynthetic pathway (Holton and Cornish 1995). With a few exceptions, all anthocyanins are produced from three precursor molecules: pelargonidin, cyanidin, and delphinidin (Grotewold 2006). There are six enzymatic reactions required for the production of anthocyanins and many also serve as branching points for the production of flavonoids, lignins, flavonols, and other phenolics that have roles in stress response and physiology (Winkel-Shirley 2002; Tahara 2007). We identified four homologs of anthocyanin biosynthetic pathway enzymes as network hubs: flavonoid 3'-

617 hydroxylase ( $F 3$ ' $H$; cluster8703_1rr.inclade1.ortho3; module ME12), dihydroflavonol 4618 reductase (DFR; cluster5082_1rr.inclade1.ortho6; module ME48), flavonoid 3',5'-

619 methyltransferase (FAOMT; cluster11783_1rr.inclade1.ortho1.tre; module ME32), and flavonol 3-O-glucosyltransferase (UFOG; cluster8241_2rr.inclade1.ortho2.tre; module ME1).

621

622 These 4 enzymes are involved in distinct steps during anthocyanin production and chemical modification in floral tissue. Activity of $F 3^{\prime} H$ directs the pathway toward the production of cyanidins and quercetin (Grotewold 2006). Cyanidin can accumulate in floral tissue as a purple-hued pigment, while quercetin plays a large role in various stress-related physiological functions (Pollastri and Tattini 2011), including response to high light and UVB radiation (Gerhardt et al. 2008) and antioxidant properties (Rice-Evans et al. 1996). Therefore, $F 3^{\prime} H$ as an important hub in the networks makes sense given its enormous pleiotropic role in various physiological responses. Just prior to the production of anthocyanins, $D F R$ catalyzes the reaction to produce pelargonidins, cyanidins, and delphinidins from the precursor molecules dihydrokaempferol, dihydroquercetin, and dihyrdomyricetin, respectively (Grotewold 2006). Shifts in DFR expression, function, and substrate specificity have been implicated in many evolutionary transitions in flower color (Smith et al. 2013; Wu et al. 2013).

Further modifications to anthocyanins, such as methylation, glycosylation, and acylation, produce a wide variety of compounds and hues (Wessinger and Rausher 2012). We identified homologs to both a methyltransferase (FAOMT) and glucosyltransferase (UFOG) as hubs in the networks. Methylation of anthocyanins have been widely studied in petunia (Provenzano et al. 2014), grapes (Hugueney et al. 2009) and tree peony (Du et al. 2015), demonstrating that methylation can impact the hue, either shifting it more toward purple (Sakata et al. 1995) or 
639 sometimes red (Tanaka et al. 2008). Glycosylation stabilizes anthocyanins molecules for 640 subsequent transfer to acidic vacuoles (Saito and Yamazaki 2002), increasing their solubility, 641 and influencing their color variability by inter- and intra-molecular stacking (Springob et al. 642 2003). Finding homologs of FAOMT and UFOG as network hubs reflect the importance of 643 anthocyanin modification for different flower color hues among Achimenes species.

644 Carotenoid biosynthesis - Carotenoids are red, orange, and yellow pigments that are 645 produced in plastids and are essential for photosynthesis, but also accumulate as secondary 646 metabolites in flowers to attract pollinators (Kevan and Baker 1983). Floral and fruit carotenoids 647 can also be used to produce volatile apocarotenoids, which may further enhance plant-animal 648 interactions during pollination and seed dispersal (Dudareva et al. 2006). They may also serve as 649

650 precursors to the production of the hormone abscisic acid and strigalactones that are involved in many plant developmental processes (Rosas-Saavedra et al. 2016). We identified 4 homologs of

651

652

653

654

655

656

657

658

659

660

661

662

663

664

665

666

667

668

669

670

671

672

673

674

675

676

677

678 carotenoid biosynthetic pathway enzymes as network hubs: geranylgeranyl pyrophosphate synthase (GGPPS; cluster10945_2rr.inclade1.ortho1.tre; module ME35), phytoene dehydrogenase (PDS; cluster13048_1rr.inclade1.ortho1.tre; module ME4), lycopene beta cyclase (LCYB; cluster13774_1rr.inclade1.ortho1.tre; module ME7), and capsanthin-capsorubin synthase (CCS; cluster13583_1rr.inclade1.ortho2.tre; module ME6).

Each of these 4 enzymes catalyzes reactions during different steps of carotenoid production. GGPPS produces the main precursor molecular geranylgeranyl pyrophosphate (GGPP) to carotenoid biosynthesis that is produced as the end-product of the plastid methylerythritol 4-phosphate (MEP) pathway. Two GGPP molecules are then condensed into phytoene and undergoes four successive dehydrogenations and isomerizations to produce lycopene. $P D S$ catalyzes the first two desaturation steps that transform the colorless phytoene into the red-colored lycopene. $P D S$ is a rate-limiting enzyme in carotenoid biosynthesis (Chamovitz et al. 1993) and down-regulation has been shown to lead to large accumulations of phytoene (Busch et al. 2002) and decreases in total carotenoid, chlorophyll, and photosynthetic efficiency (Wang et al. 2009).

Lycopene is the main starting compound for a large variety of carotenoids. $L C Y B$ takes lycopene as a substrate and subsequently produces beta-carotene. Many plants use tissue-specific isoforms of $\angle C Y B$ genes that are expressed in fruits or flowers that often correlate with the accumulation of beta-carotene or downstream xanthophylls (Ronen et al. 2000; Ahrazem et al. 2010; Devitt et al. 2010). Beta-carotenes are hypothesized to be the primary carotenoids in Achimenes flowers (Roberts and Roalson 2017). Another functionally related carotenoid cyclase enzyme is $C C S$ (or neoxanthin synthase, $N S Y$ ) which catalyzes the final step in the carotenoid pathway and converts the yellow-colored violaxanthin into neoxanthin (Parry and Horgan 1991). Both violaxanthin and neoxanthin can be further used for producing the plant hormone abscisic acid (Neuman et al. 2014). Both enzymes are likely functionally important in the developing flowers for the biosynthesis of beta-carotene and xanthophylls.

Flower development-Very few studies have so far investigated the molecular mechanisms involved in the development of flower shape in the Gesneriaceae (Gao et al. 2008; 
679 Zhou et al. 2008; Alexandre et al. 2015). However, extensive work has been done in model

680 organisms, such as Arabidopsis thaliana and Antirrhinum majus, which can provide insight into

681 the potential roles that many transcription factors might play in determining floral organ identity

682 and shape in Achimenes. Among the network hubs, we identified several homologs that are likely

683 involved in floral organ development, including mixta (MIXTA;

684 cluster8699_1rr.inclade1.ortho3.tre; modules ME5 and ME8), agamous ( $A G$;

685 cluster7311_1rr.inclade1.ortho3.tre; module ME23), globosa (GLO;

686 cluster2750_1rr.inclade1.ortho3.tre; module ME37), and tcp4 (TCP4;

687 cluster12380_1rr.inclade1.ortho2.tre; module ME22).

688 Petal identity and petal surface morphology are both thought to be important components 689 for pollinator attraction (Noda et al. 1994; Whitney et al. 2011). MIXTA was first characterized

690 from Antirrhinum and controls the development of the conical cell shape in the petal epidermis

691 (Noda et al. 1994). Consequently, MIXTA and MIXTA-like genes have been characterized in 692 other model systems, such as Arabidopsis and Mimulus for their similar role in epidermal cell 693 morphogenesis (Baumann et al. 2007) as well as trichome development (Gilding and Marks 694 2010; Scoville et al. 2011). The homolog of MIXTA in our study was a hub node in both 695 networks, suggesting it might play an important role throughout flower development in 696 determining petal surface morphology.

697 Early floral organ determination requires the involvement of several MADS-box 698 transcription factors, such as $A G$ and $G L O$. We identified homologs of $A G$ as a hub in the Bud 699 stage and $G L O$ as a hub in the D stage (Supplemental Table 7). $A G$ has been shown in

700

701

702

703

704

705

706

707

708

709

710

711

712

713

714

715

716

717

718 Arabidopsis to be expressed in early flower development in order to terminate meristem activity and promote the development of stamens and carpels, while working in combination with APETALA3, PISTILLATA, and SEPALLATA (Gómez-Mena et al. 2005). More recently homologs of $A G$ were demonstrated to be involved in nectary development in both Arabidopsis and Petunia (Morel et al. 2018), another floral trait important for plant-pollinator interaction. GLO was first characterized in Antirrhinum and Arabidopsis and is also involved with stamen identity, as well as petal identity (Tröbner et al. 1992; Goto and Meyerowitz 1994). Outside of these model systems, its expression patterns and role in the evolution of complex floral forms have been examined in numerous plant lineages, from monocots (Bartlett and Specht 2010) to Solanaceae (Zhang et al. 2014). $A G$ and $G L O$ will be interesting candidates to begin exploring further how petals, stamens, carpels, and nectary development in Achimenes.

Lastly, the homolog of TCP4 was identified as a hub during the Bud stage, and may be important for its potential role in petal development and cell proliferation. Looking into the genetic factors regulating the elaboration of petal growth will be important for understanding how corolla spurs develop in Achimenes (Figure 1). The role of TCP4 been characterized in Arabidopsis for its role in both petal growth, showing restricted expression to the developing petal tissue (Nag et al. 2009). More recent work using gene regulatory networks in Arabidopsis has shown that petal size is controlled by a SEPALLATA3-regulated miR319/TCP4 module (Chen et al. 2018). Outside of Arabidopsis, TCP4 was also implicated for a having a role in the 
719 development of the petal spur in Aquilegia (Yant et al. 2015). Therefore, considering both the 720 role for TCP4 in petal and spur development demonstrated in Arabidopsis and Aquilegia and its

721

722

723

724

725

726

727

728

729

730

731

732

733

734

735

736

737

738

739

740

741

742

743

744

745

746

747

748

749

750

751

752

753

754

755

756

757

758 identification as a hub gene in our networks, we think this gene requires further interrogation.

Peripheral genes are enriched for transcriptional regulators in later stages of development Peripheral nodes have low connectivity within co-expression networks (Supplemental Table 7), tend to have higher rates of molecular evolution (Masalia et al. 2017), and have been hypothesized to contribute more to evolutionary innovations than network hubs (Ichihashi et al. 2014). Of the 951 nodes we defined as peripheral in both Bud and D stage networks, roughly a third were consistently peripheral under our definitions (lowest 10\% connected; Supplemental Table 7). After identifying the nodes with homologies to transcriptional regulators, we found there was no difference in the expected number in the early Bud stage, while there were greater than expected numbers of transcriptional regulators in the periphery of the D stage (see Results). No such patterns existed when we tested these transcriptional regulators as hub genes. While Mähler et al. (2017) found an enrichment for transcription factors among hub genes in a single species network of Populus tremula, our results in a multi-species network find the opposite pattern. Shifting the expression patterns of various genes and pathways can lead to phenotypic divergence among closely related species (West-Eberhard 1989). This can be seen during later stages of flower development when distinct traits, such as red or purple flower color, can appear in different species due to shifting expression patterns within the anthocyanin biosynthetic pathway (Smith and Rausher 2011). The connection between the apparent overabundance of transcriptional regulators in later stages of flower development and the rapid phenotypic divergence in floral form will need to be explored further.

Several notable peripheral nodes were identified for their potential involvement in important floral processes (Supplemental Table 7). Three homologs were identified in the periphery of the Bud stage with potential involvement in the determination of zygmorphic (bilateral) floral symmetry: two homologs of divaricata $(D I V$;

cluster13684_1rr.inclade1.ortho1.tre, cluster8585_1rr.inclade1.ortho1.tre; ME26 and ME0) and one homolog of dichotoma (DICH; cluster13245_1rr.inclade1.ortho1.tre; ME0). Bilateral symmetry has been proposed as a mechanism that facilitates pollen transfer by insects (Galliot et al. 2006). Both $D I V$ and $D I C H$ are TCP transcription factors that were first characterized in Antirrhinum (Almeida et al. 1997; Luo et al. 1996), and show localized expression to the ventral and dorsal portions of the petal, respectively. Achimenes flowers at the Bud stage are lacking or only just beginning to establish floral asymmetry and the relegation of both $D I V$ and $D I C H$ to the network periphery may reflect this small role. The role of these genes has not been studied in the Gesneriaceae, but have been examined more recently in other non-model systems for their role in the evolution of pollination syndromes (Zhang et al. 2010; Preston et al. 2011). Additionally, a homolog of the homeobox wuschel gene (WUS; cluster9273_1rr.inclade1.ortho1.tre; module ME0) was identified from the periphery of the D stage network. WUS was first characterized in Arabidopsis for its role in meristem cell maintenance (Laux et al. 1996) and its expression is

Peer) reviewing PDF | (2019:10:42302:1:1:NEW 10 Feb 2020) 
759 localized only to the meristem (Mayer et al. 1998). Once the floral meristem has been initiated, $760 W U S$ starts to be repressed by $A G$ (hub gene, described above) (Sun et al. 2009). The relegation

761 of WUS to the periphery in our networks at the D stage might then be expected given that it plays

762 such a prominent role during early floral development and the vegetative to reproductive

763 transition.

764

765

766

767

768

769

770

771

772

773

774

\section{Evolutionary rates are correlated to network connectivity}

Increased protein evolutionary rates during rapid diversification has been suggested in other radiations of animals and plants (Kapralov et al. 2013; Brawand et al. 2014; Pease et al. 2016). Our study is among the first to examine patterns of network evolution across multiple closely-related species of plants with multiple transitions in flower type and pollination syndrome. Hummingbirds are hypothesized to have had an important influence on diversification in Neotropical Gesneriaceae (Roalson and Roberts 2016; Serrano-Serrano et al. 2017b), while the role of butterflies has not been explored. Modules correlated with hummingbird or butterfly pollination syndromes did not exhibit clear increases or decreases in evolutionary rates compared to other modules (Supplemental Table 9). Detecting selection is subject to many factors, such as

775 a gene's transcriptional abundance and its importance within the protein interaction network

776 (Lemos et al. 2005). For instance, we found that higher network connectivity was strongly

777

778

779

780

781

782

783

784

785

786

787

788

789

790

791

792

793

794

795

796

797

798 associated with lower evolutionary rates across both networks in our study (Figure 7; Supplemental Table 9). This corroborates the recent results found in other eukaryotic systems (Morandin et al. 2016; Masalia et al. 2017; Josephs et al. 2017; Mähler et al. 2017) while also showing the apparent strength of correlation between connectivity and evolutionary rate differs between studies and systems.

Network effects (such as the type of network analysis employed) and the expression levels of highly abundant genes are known to affect evolutionary rates, potentially confounding analysis of selection pressure (Krylov et al. 2003). We found that $d_{\mathrm{N}} / d_{\mathrm{S}}$ in early development may be dependent on both connectivity and expression levels, while this interactive effect was gone during later development (Supplemental Table 9). As there are few highly connected genes (hubs, which are important determinants of the observed network structure), a random mutation would be less likely to affect such a gene. Changing the amino acid sequence of a hub gene could have multiple associated effects, some deleterious (Hahn and Kern 2004; Luisi et al. 2015). In comparison, a random mutation would be more likely to affect a gene with lower connectivity, while also resulting in variants with fewer negative consequences. The hub genes have more direct interactions and are more likely to be essential and involved in more biological processes, placing them under greater constraint.

\section{Numerous network modules correlated to floral form}

One primary use for gene co-expression network analyses is to identify modules of coexpressed genes whose overall expression (as measured by the eigengene) may correlate with different traits. These traits can be both qualitative or quantitative, such as social caste (Morandin 
799

800

801

802

803

804

805

806

807

808

809

810

811

812

813

814

815

816

817

818

819

820

821

822

823

824

825

826

827

828

829

830

831

832

833

834

835

836

837

838

et al. 2016), seed oil production (Hu et al. 2016), or anther development (Hollender et al. 2014). While we tested only qualitative traits here, future studies could also correlate eigengene expression to quantitative variables such as nectary size or sucrose concentration, since these appear to be another important component of pollination syndrome delimitation (Perret et al. 2001; Katzer et al. 2019; Vandelook et al. 2019). Since our dataset was composed of samples from 12 species, we performed correlation analyses using a phylogenetic model to test whether module expression correlated to different flower colors, flower shapes, corolla spurs, and pollination syndromes. Nearly half of our modules in both networks had evidence for an association with these floral traits (Figures 5-6). There were no modules whose correlation to a pollination syndrome had increased eigengene expression in all species sharing that trait (Figures 5-6). Instead many modules with correlation to a trait showed a phylogenetic pattern with a shared increased eigengene expression among the most closely related species. For example, module ME23 in the Bud stage network had a correlation to butterfly pollination and increased eigengene expression in A. cettoana and A. longiflora, both members of Clade 1 (Figure 2). Our aim was to identify sets of co-expressed genes that would provide candidates for involvement in the development of these traits.

For instance, many of the candidate hubs we identified (see above) were found in modules that were correlated to one or multiple traits to which they might contribute, such as PHAN (module ME4, correlated to corolla spurs) and DFR (module ME48, correlated to hummingbird pollination and purple flowers) (Supplemental Table 2). Additionally, modules correlated to various floral traits were enriched for multiple genes that might be involved in the development of those traits (Supplemental Table 8). Module ME53 was correlated to the corolla spurs found in $A$. grandiflora and A. patens in the D stage network and was enriched for many genes involved in cell division and cell growth (Figure 6; Supplemental Table 4). Other times, modules were correlated to a specific trait but the enriched GO terms did not provide a clear idea of how these co-expressed orthogroups may contribute (Supplemental Tables 3-4). In our analyses, we considered relatively few traits and only focused on those that have traditionally been considered the most important for the differentiation of floral form in Achimenes, namely flower color, flower shape, and corolla spurs. Undoubtedly there are a myriad number of molecular and biochemical pathways that we have not considered here that underlie the development of these complex floral phenotypes. With the idea that networks represent complex systems of gene and protein interactions, it may be that these seemingly simple and generalized floral phenotypes are produced through much more complex means than many have previously considered.

\section{Strengths and limitations of network analyses in non-model organisms}

Our analysis covers genes with orthologs found in most species, representing a set of evolutionarily conserved genes. While no reference genome currently exists for Neotropical Gesneriaceae, we constructed, annotated, and presented de novo transcriptomes for 10 Achimenes species as well as 2 outgroup species, E. verticillata and G. cuneifolia. Our approach 
839 enables functional genomic studies in this non-model system, but the potential for misassemblies 840 may bias our downstream results (Hornett and Wheat 2012). Using a gene tree-based approach to 841 carefully identify orthogroups, we focused our attention on conserved gene regulatory machinery 842 and not on taxonomically restricted genes. The latter may be involved in species-specific

843 functions. Nevertheless, it is worth noting that many recent studies have indicated that transitions 844 in floral form may be due to the shifting expression of genes found in conserved genetic 845 pathways (Wessinger and Rausher 2014). Taxonomically restricted genes are likely interacting 846 with existing regulatory pathways, and the manner in which they integrate into the co-expression 847 modules identified here will be a fascinating topic for future research. With extended sampling within Achimenes we could infer species-specific co-expression networks and perform more detailed cross-species comparisons. We expect that these taxonomically restricted genes may be

850 poorly connected with the network, which may allow for increased diversification.

We characterized gene expression patterns by sequencing RNA across two timepoints during flower development. Previous gene expression experiments across three timepoints (early Bud, intermediate D, and late Pre-anthesis stages) in four Achimenes species suggested that the majority of differential gene expression occurred between the early Bud stage and the intermediate D stage (Roberts and Roalson 2017). We believe our approach allows us to capture dynamic transcriptional patterns during flower development for comparison across multiple species. We found that the co-expression networks for the Bud and D stages were largely preserved, despite there being a few stage specific modules (Supplemental Table 5). However, this approach may greatly over-simplify the nature of transcriptional regulation in these species. Organ- and structure-specific expression studies may have greater power to detect floral form-

862

863 specific differences, both in terms of the number of differentially expressed genes, and coexpression network structure (Hollender et al. 2014; Suzuki et al. 2017; Shahan et al. 2018). As a result, our data likely represents an underestimate of the true number of modules. Future studies could focus on the comparative analysis of expression patterns in specific organs, such as the petals, stigma, and stamens, and additional timepoints, which would provide greater functional insight into the evolution, development, and diversification of these organs. analyses because it can capture system-level properties (Langfelder and Horvath 2008). Most phenotypes involve interactions of proteins from diverse biochemical pathways. Although inferred modules do not necessarily correspond to entire biochemical pathways, or other components of cellular organization, the approach performs well in reconstructing the complexity of protein-protein interaction networks (Allen et al. 2012). In many systems this approach has succeeded in identifying candidate genes for various biological traits, for example floral organ development in strawberry (Hollender et al. 2014; Shahan et al. 2018) or carotenoid accumulation in carrots (Iorizzo et al. 2016). Our results demonstrate the difficulty in identifying gene clusters that underlie highly correlated floral traits, especially in non-model systems, as seen by the overlapping correlation of modules associated with flower shape and color (Figures 5-6). It has been shown in many plant systems that floral traits tend to be selected together by 
879 pollinators (Cuartas-Domínguez and Medel 2010; Sletvold et al. 2010), making it challenging to 880 separate correlated modules from causal modules. Co-expression networks are foundationally 881 about correlations between genes (van Dam et al. 2018), making them useful in identifying novel 882 sets of genes that may be functionally relevant for trait of interest. Other approaches, such as 883 quantitative trait locus (QTL) analyses may provide more promise in identifying distinct genes 884 that are involved in the divergence of floral traits. QTL studies have already shown promise in 885 identifying genetic loci that underlie pollination syndrome divergence in Mimulus (Yuan et al. 886 2013), Penstemon (Wessinger et al. 2014), and Rhytidophyllum (Alexandre et al. 2015). We were able to identify numerous candidate orthogroups involved in the development of

889

890

891

892

893

894

895

896

897

898

899

900

901

902

903

904

905

906

907

908

909

910

911

912

913

914

915

916

917

918

floral form in our non-model system. While no functional genomic data exists yet for Achimenes, our annotations are based on the manually curated SwissProt database. De novo assemblies provide important data for non-model organisms, but there can be biases introduced during assembly and annotation due to potentially missing genes (Hornett and Wheat 2012). We attempted to limit this bias by focusing only on shared orthogroups found in the majority (at least 6) of our sampled Achimenes. Applying this network-based approach to the diversification of flowers across multiple species in Achimenes, we recovered distinct modules of co-expressed genes that may underlie a range of floral phenotypic traits tied to different pollination syndromes. Although our data come from whole flowers during development, and do not allow us to test specific hypotheses regarding whether particular genes have shifted expression location in the developing flower, they do suggest that there are conserved regulatory modules that may have been co-opted in convergent flower types multiple times. It will be interesting to apply network analyses to study floral diversification in additional lineages and examine how conserved or divergent the patterns are across angiosperms.

\section{Conclusions}

Floral forms corresponding to bee, butterfly, and hummingbird pollination have evolved multiple times across the Neotropical plant genus Achimenes. Genome-wide gene expression estimates were taken from flowers in 12 species across two development stages in order to construct, analyze, and compare stage-specific gene co-expression networks. We hypothesized that numerous modules in each network would correlate to these pollination syndromes and that central genes in the network may be candidates for involvement in the development of important floral traits, such as flower color and shape. We found that nearly a third of modules were correlated to the pollination syndromes and many more were correlated to different flower colors and shapes. The outgroup species, E. verticillata (bee pollinated) and G. cuneifolia (hummingbird pollinated), displayed correlation patterns similar to the ingroup species that shared floral traits, suggesting that some co-expression patterns might be shared across evolutionary distances. Several of the hub genes in the networks were homologs of the anthocyanin and carotenoid biosynthetic pathways, important for the production of floral pigments that attract different pollinators. A negative relationship between network connectivity and $d_{\mathrm{N}} / d_{\mathrm{S}}$ corroborates the findings in model systems that more centrally located nodes (likely 
919 hubs) are under increased evolutionary constraint. We found that the less connected genes 920 (peripheral) are under more relaxed constraints and contained numerous transcriptional 921 regulators. Our results demonstrate the utility of applying co-expression network analyses in 922 non-model plant lineages to begin identifying important modules and pathways that will be 923 useful starting points for additional analyses into the evolution and development of floral form.

924

925

926 Acknowledgements

927

928

929

930

931

932

933

934

935

936

937

938

939

940

941

942

943

944

945

946

947

948

949

950

951

952

953

954

955

956

957

958

We thank Joanna Kelley, Amit Dhingra, and Andrew McCubbin for helpful comments that improved this work. Sequencing was performed at the Genomics Core Lab at Washington State University, Spokane, and the Genomics Sequencing and Analysis Facility at the University of Texas, Austin.

\section{References}

Ahrazem O, Rubio-Moraga A, López RC, Gómez-Gómez L. 2010. The expression of a chromoplast-specific lycopene beta cyclase gene is involved in high production of saffron's apocarotenoid precursors. J Exp Bot 61:105-119.

Alexa A, Rahnenführer J, Lengauer T. 2006. Improved scoring of functional groups from gene expression data by decorrelating GO graph structure. Bioinformatics 22:1600-1607.

Alexandre H, Vrignaud J, Mangin B, Joly S. 2015. Genetic architecture of pollination syndrome transition between hummingbird-specialist and generalist species in the genus Rhytidophyllum (Gesneriaceae). PeerJ 3:e1028.

Allen JD, Xie Y, Chen M, Girard L, Xiao G. 2012. Computing statistical methods for constructing large scale gene networks. PLOS One 7:e29348.

Almeida J, Rocheta M, Galego L. 1997. Genetic control of flower shape in Antirrhinum majus. Development 124:1387-1392.

Amorim FW, Galetto L, Sazima M. 2013. Beyond the pollination syndrome: nectar ecology and the role of diurnal and nocturnal pollinators in the reproductive success of Inga sessilis. Plant Biol 15:317-327.

Baumann K, Perez-Rodriguez M, Bradley D, Venail J, Bailey P, Jin H, Koes R, Roberts K, Martin C. 2007. Control of cell and petal morphogenesis by R2R3 MYB transcription factors. Development 134:1691-1701.

Barrett SC. 2013. The evolution of plant reproductive systems: how often are transitions irreversible? P Roy Soc B-Biol Sci 280: 20130913.

Bartlett ME, Specht CD. 2010. Evidence for the involvement of GLOBOSA-like gene duplications and expression divergence in the evolution of floral morphology in the Zingiberales. New Phytol 187:521-541.

Bolger AM, Lohse M, Usadel B. 2014. Trimmomatic: a flexible trimmer for Illumina sequence data. Bioinformatics 30:2114-2120.

Bolstad B. 2018. preprocessCore: A collection of pre-processing functions. R package version 1.46.0, https://github.com/bmbolstad/preprocessCore. 
959 Bradshaw HD, Otto KG, Frewen BE, McKay JK, Schemske DW. 1998. Quantitative trait loci

960

961

962

963

964

965

966

967

968

969

970

971

972

973

974

975

976

977

978

979

980

981

982

983

984

985

986

987

988

989

990

991

992

993

994

995

996

997

998 affecting differences in floral morphology between two species of monkeyflower (Mimulus). Genetics 149:367-382.

Brawand D, Soumillon M, Necsulea A, Julien P, Csárdi G, Harrigan P, Weier M, Liechti A, Aximu-Petri A, Kircher M, Albert FW. 2011. The evolution of gene expression levels in mammalian organs. Nature 478:343.

Brawand D, Wagner CE, Li YI, Malinsky M, Keller I, Fan S, Simakov O, Ng AY, Lim ZW, Bezault E, Turner-Maier J. 2014. The genomic substrate for adaptive radiation in African cichlid fish. Nature 513:375.

Bray NL, Pimentel H, Melsted P, Pachter L. 2016. Near-optimal probabilistic RNA-seq quantification. Nat Biotechnol 34:525.

Buchfink B, Xie C, Huson DH. 2014. Fast and sensitive protein alignment using DIAMOND. Nat Methods 12:59-60.

Busch M, Seuter A, Hain R. 2002. Functional analysis of the early steps of carotenoid biosynthesis in tobacco. Plant Physiol 128:439-453.

Chamovitz D, Sandmann G, Hirschberg J. 1993. Molecular and biochemical characterization of herbicide-resistant mutants of cyanobacteria reveals that phytoene desaturation is a ratelimiting step in carotenoid biosynthesis. J Biol Chem 268:17348-17353.

Chen D, Yan W, Fu LY, Kaufmann K. 2018. Architecture of gene regulatory networks controlling flower development in Arabidopsis thaliana. Nat Commun 9:1-13.

Cortés-Flores J, Hernández-Esquivel KB, González-Rodríguez A, Ibarra-Manríquez G. 2017. Flowering phenology, growth forms, and pollination syndromes in tropical and dry forest species: influence of phylogeny and abiotic factors. Am J Bot 104:39-49.

Cronk Q, Ojeda I. 2008. Bird-pollinated flowers in an evolutionary and molecular context. J Exp Bot 59:715-727.

Cuartas-Domínguez M, Mendel R. 2010. Pollinator-mediated selection and experimental manipulation of the flower phenotype in Chloraea bletioides. Funct Ecol 24:1219-1227.

Des Marais DL, Rausher MD. 2010. Parallel evolution at multiple levels in the origin of hummingbird pollination flowers in Ipomoea. Evolution 64:2044-2054.

De Smet R, Adams KL, Vandepoele K, Van Montagu MC, Maere S, Van de Peer Y. 2013. Convergent gene loss following gene and genome duplications creates single-copy families in flowering plants. P Natl Acad Sci USA 110:2898-2903.

Devitt LC, Fanning K, Dietzgen RG, Holton TA. 2010. Isolation and functional characterization of a lycopene $\beta$-cyclase gene that controls fruit colour of papaya (Carica papaya L.). $\mathrm{J}$ Exp Bot 61:33-39.

Du H, Wu J, Ji KX, Zeng QY, Bhuiya MW, Su S, Shu QY, Ren HX, Liu ZA, Wang LS. 2015. Methylation mediated by an anthocyanin O-methyltransferase is involved in purple flower coloration in Paeonia. J Exp Bot 66:6563-6577.

Dudareva N, Negre F, Nagegowda DA, Orlova I. 2006. Plant volatiles: recent advances and future perspectives. Cr Rev Plant Sci 25:417-440.

Peer) reviewing PDF | (2019:10:42302:1:1:NEW 10 Feb 2020) 
999 Enright AJ, Van Dongen S, Ouzounis CA. 2002. An efficient algorithm for large-scale detection 1000 of protein families. Nucleic Acids Res 30:1575-1584.

1001 Faegri K, van der Pijl L. 1979. Principles of Pollination Ecology. Oxford: Pergamon Press.

1002 Fenster CB, Armbruster WS, Wilson P, Dudash MR, Thomson JD. 2004. Pollination syndromes

1003

1004 and floral specialization. Annu Rev Ecol Evol S 35:375-403.

1005

Galliot C, Stuurman J, Kuhlemeier C. 2006. The genetic dissection of floral pollination syndromes. Curr Opin Plant Biol 9:78-82.

1006

Gao Q, Tao JH, Yan D, Wang YZ, Li ZY. 2008. Expression differentiation of CYC-like floral

1007

1008 symmetry genes correlated with their protein sequence divergence in Chirita heterotricha

1009 (Gesneriaceae). Dev Genes Evol 218:341-351.

1010

Gelman A, Rubin DB. 1992. Inference from iterative simulation using multiple sequences. Stat

1011 Sci 7:457-472.

Gerhardt KK, Lampi MA, Greenberg BM. 2008. The effect of far-red light on plant growth and

1012

1013

1014 flavonoid accumulation in Brassica napus in the presence of ultraviolet B radiation. Photochem Photobiol 84:1445-1454.

1015

1016

1017

1018

1019

1020

1021

1022

1023

1024

1025

1026

1027

1028

1029

1030

1031

1032

1033

1034

1035

1036

1037

Gervasi DD, Schiestl FP. 2017. Real-time divergent evolution in plants driven by pollinators. Nat Commun 8: 14691.

Gilding EK, Marks MD. 2010. Analysis of purified glabra3-shapeshifter trichomes reveals a role for NOECK in regulating early trichome morphogenic events. Plant J 64:304-317.

Gómez-Mena C, De Folter S, Costa MMR, Angenent GC, Sablowski R. 2005. Transcriptional program controlled by the floral homeotic gene AGAMOUS during early organogenesis. Development 132:429-438.

Goto K, Meyerowitz EM. 1994. Function and regulation of the Arabidopsis floral homeotic gene PISTILLATA. Gene Dev 8:1548-1560.

Grabherr MG, Haas BJ, Yassour M, Levin JZ, Thompson DA, Amit I, Adiconis X, Fan L, Raychowdhury R, Zeng Q, Chen Z. 2011. Trinity: reconstructing a full-length transcriptome without a genome from RNA-Seq data. Nat Biotechnol 29:644.

Grotewold E. 2006. The genetics and biochemistry of floral pigments. Annu Rev Plant Biol 57:761-780.

Guillerme T, Healy K. 2014. mulTree: a package for running MCMCglmm analysis on multiple trees. ZENODO. 10.5281/zenodo.12902.

Hahn MW, Kern AD. 2004. Comparative genomics of centrality and essentiality in three eukaryotic protein-interaction networks. Mol Biol Evol 22:803-806.

Haas BJ, Papanicolaou A, Yassour M, Grabherr M, Blood PD, Bowden J, Couger MB, Eccles D, Li B, Lieber M, MacManes MD. 2013. De novo transcript sequence reconstruction from RNA-seq using the Trinity platform for reference generation and analysis. Nat Protoc 8:1494.

Hadfield JD. 2010. MCMC methods for multi-response generalized linear mixed models: the MCMCglmm R pckage. J Stat Softw 33:1-22. 
1038 Hermann K, Klahre U, Venail J, Brandenburg A, Kuhlemeier C. 2015. The genetics of

1039

1040

1041

1042

1043

1044

1045

1046

1047

1048

1049

1050

1051

1052

1053

1054

1055

1056

1057

1058

1059

1060

1061

1062

1063

1064

1065

1066

1067

1068

1069

1070

1071

1072

1073

1074

1075

1076

reproductive organ morphology in two Petunia species with contrasting pollination syndromes. Planta 241:1241-1254.

Hoballah ME, Gübitz T, Stuurman J, Broger L, Barone M, Mandel T, Dell'Olivo A, Arnold M, Kuhlemeier C. 2007. Single gene-mediated shift in pollinator attraction in Petunia. Plant Cell 19: 779-790.

Hollender CA, Kang C, Darwish O, Geretz A, Matthews BF, Slovin J, Alkharouf N, Liu Z. 2014. Floral transcriptomes in woodland strawberry uncover developing receptacle and anther gene networks. Plant Physiol 165:1062-1075.

Hornett EA, Wheat CW. 2012. Quantitative RNA-Seq analysis in non-model species: assessing transcriptome assemblies as a scaffold and the utility of evolutionary divergent genomic reference species. BMC Genomics 13:361.

Horvath S, Dong J. 2008. Geometric interpretation of gene co-expression network analysis. PLOS Comput Biol 4:e1000117.

Howarth DG, Martins T, Chimney E, Donoghue MJ. 2011. Diversification of CYCLOIDEA expression in the evolution of bilateral flower symmetry in Caprifoliaceae and Lonicera (Dipsacales). Ann Bot-London 107:1521-1532.

Hu G, Hovav R, Grover CE, Faigenboim-Doron A, Kadmon N, Page JT, Udall JA, Wendel JF. 2016. Evolutionary conservation and divergence of gene coexpression networks in Gossypium (cotton) seeds. Genome Biol Evol 8:3765-3783.

Hugueney P, Provenzano S, Verriès C, Ferrandino A, Meudec E, Batelli G, Merdinoglu D, Cheynier V, Schubert A, Ageorges A. 2009. A novel cation-dependent Omethyltransferase involved in anthocyanin methylation in grapevine. Plant Physiol 150:2057-2070.

Ichihashi Y, Aguilar-Martínez JA, Farhi M, Chitwood DH, Kumar R, Millon LV, Peng J, Maloof JN, Sinha NR. 2014. Evolutionary developmental transcriptomics reveals a gene network module regulating interspecific diversity in plant leaf shape. P Natl Acad Sci USA 111:E2616-E2621.

Iorizzo M, Ellison S, Senalik D, Zeng P, Satapoomin P, Huang J, Bowman M, Iovene M, Sanseverino W, Cavagnaro P, Yildiz M. 2016. A high-quality carrot genome assembly provides new insights into carotenoid accumulation and asterid genome evolution. Nat Genet 48:657.

Josephs EB, Wright SI, Stinchcombe JR, Schoen DJ. 2017. The relationship between selection, network connectivity, and regulatory variation within a population of Capsella grandiflora. Genome Biol Evol 9:1099-1109.

Katoh K, Standley DM. 2013. MAFFT multiple sequence alignment software version 7: improvements in performance and usability. Mol Biol Evol 30:772-780.

Katzer AM, Wessinger CA, Hileman LC. 2019. Nectary size is a pollination syndrome trait in Penstemon. New Phytol 223:377-384.

PeerJ reviewing PDF | (2019:10:42302:1:1:NEW 10 Feb 2020) 
1077 Kapralov MV, Votintseva AA, Filatov DA. 2013. Molecular adaptation during a rapid adaptive

1078

1079

1080

1081

1082

1083

1084

1085

1086

1087

1088

1089

1090

1091

1092

1093

1094

1095

1096

1097

1098

1099

1100

1101

1102

1103

1104

1105

1106

1107

1108

1109

1110

1111

1112

1113

1114

1115

1116 radiation. Mol Biol Evol 30:1051-1059.

Kevan PG, Baker HG. 1983. Insects as flower visitors and pollinators. Annu Rev Entomol 28:407-453.

Krylov DM, Wolf YI, Rogozin IB, Koonin EV. 2003. Gene loss, protein sequence divergence, gene dispensability, expression level, and interactivity are correlated in eukaryotic evolution. Genome Res 13:2229-2235.

Langfelder P, Horvath S. 2008. WGCNA: an R package for weighted correlation network analysis. BMC Bioinformatics 9:559.

Langfelder P, Luo R, Oldham MC, Horvath S. 2011. Is my network module preserved and reproducible? PLOS Comput Biol 7:e1001057.

Laux T, Mayer KF, Berger J, Jurgens G. 1996. The WUSCHEL gene is required for shoot and floral meristem integrity in Arabidopsis. Development 122:87-96.

Lemos B, Bettencourt BR, Meiklejohn CD, Hartl DL. 2005. Evolution of proteins and gene expression levels are coupled in Drosophila and are independently associated with mRNA abundance, protein length, and number of protein-protein interactions. Mol Biol Evol 22:1345-1354.

Li W, Godzik A. 2006. Cd-hit: a fast program for clustering and comparing large sets of protein or nucleotide sequences. Bioinformatics 22:1658-1659.

Lohmann JU, Hong RL, Hobe M, Busch MA, Parcy F, Simon R, Weigel D. 2001. A molecular link between stem cell regulation and floral patterning in Arabidopsis. Cell 105:793-803.

Love MI, Huber W, Anders S. 2014. Moderated estimation of fold change and dispersion for RNA-seq data with DESeq2. Genome Biol 15:550.

Luisi P, Alvarez-Ponce D, Pybus M, Fares MA, Bertranpetit J, Laayouni H. 2015. Recent positive selection has acted on genes encoding proteins with more interactions within the whole human interactome. Genome Biol Evol 7:1141-1154.

Luo D, Carpenter R, Copsey L, Vincent C, Clark J, Coen E. 1996. Control of organ asymmetry in flowers of Antirrhinum. Cell 99:367-376.

Ma X, Zhao H, Xu W, You Q, Yan H, Gao Z, Su Z. 2018. Co-expression gene network analysis and functional module identification in bamboo growth and development. Front Genetics 9:574.

Mähler N, Wang J, Terebieniec BK, Ingvarsson PK, Street NR, Hvidsten TR. 2017. Gene coexpression network connectivity is an important determinant of selective constraint. PLOS Genet 13:e1006402.

Martén-Rodríguez S, Quesada M, Castrao AA, Lopezaraiza-Mikel M, Fenster CB. 2015. A comparison of reproductive strategies between island and mainland Caribbean Gesneriaceae. J Ecol 103:1190-1204.

Masalia RR, Bewick AJ, Burke JM. 2017. Connectivity in gene coexpression networks negatively correlates with rates of molecular evolution in flowering plants. PLOS One 12:e182289. 
1117 Mason MJ, Fan G, Plath K, Zhou Q, Horvath S. 2009. Signed weighted gene co-expression

1118

1119

1120

1121

1122

1123

1124

1125

1126

1127

1128

1129

1130

1131

1132

1133

1134

1135

1136

1137

1138

1139

1140

1141

1142

1143

1144

1145

1146

1147

1148

1149

1150

1151

1152

1153

1154

1155 network analysis of transcriptional regulation in murine embryonic stem cells. BMC Genomics 10:327.

Mayer KF, Schoof H, Haecker A, Lenhard M, Jürgens G, Laux T. 1998. Role of WUSCHEL in regulating stem cell fate in the Arabidopsis shoot meristem. Cell 95:805-815.

Morandin C, Tin MM, Abril S, Gómez C, Pontieri L, Schiøtt M, Sundström L, Tsuji K, Pedersen JS, Helanterä H, Mikheyev AS. 2016. Comparative transcriptomics reveals the conserved building blocks involved in parallel evolution of diverse phenotypic traits in ants. Genome Biol 17:43.

Morel P, Heijmans K, Ament K, Chopy M, Trehin C, Chambrier P, Bento SR, Bimbo A, Vandenbussche M. 2018. The floral C-lineage genes trigger nectary development in Petunia and Arabidopsis. Plant Cell 30:2020-2037.

Nag A, King S, Jack T. 2009. miR319a targeting of TCP4 is critical for petal growth and development in Arabidopsis. P Natl Acad Sci USA 106:22534-22539.

Neuman H, Galpaz N, Cunningham Jr, FX, Zamir D, Hirschberg J. 2014. The tomato mutation nxd1 reveals a gene necessary for neoxanthin biosynthesis and demonstrates that violaxanthin is a sufficient precursor for abscisic acid biosynthesis. Plant J 78:80-93.

Noda KI, Glover BJ, Linstead P, Martin C. 1994. Flower colour intensity depends on specialized cell shape controlled by a Myb-related transcription factor. Nature 369:661-664.

O'Meara BC, Smith SD, Armbruster WS, Harder LD, Hardy CR, Hileman LC, Hufford L, Litt A, Magallón S, Smith SA, Stevens PF. 2016. Non-equilibrium dynamics and floral trait interactions shape extant angiosperm diversity. P Roy Soc B-Biol Sci 283:20152304.

Papiorek S, Junker RR, Alves-dos-Santos I, Melo GA, Amaral-Neto LP, Sazima M, Wolowski M, Freitas L, Lunau K. 2016. Bees, birds and yellow flowers: pollinator-dependent convergent evolution of UV patterns. Plant Biol 18:46-55.

Paradis E, Claude J, Strimmer K. 2004. APE: analyses of phylogenetics and evolution in R language. Bioinformatics 20:289-290.

Parry AD, Horgan R. 1991. Carotenoids and abscisic acid (ABA) biosynthesis in higher plants. Physiol Plantarum 82:320-326.

Parsana P, Ruberman C, Jaffe AE, Schatz MC, Battle A, Leek JT. 2019. Addressing confounding artifacts in reconstruction of gene co-expression networks. Genome Biol 20:94.

Patterson AH, Bowers JE, Ruggmann R, Dubchak I, Grimwood J, Gundlach H, Haberer G, Hellsten U, Mitros T, Poliakov A, Schmutz J. 2009. The Sorghum bicolor genome and the diversification of grasses. Nature 457:551.

Pease JB, Haak DC, Hahn MW, Moyle LC. 2016. Phylogenomics reveals three sources of adaptive variation during a rapid radiation. PLOS Biol 14:e1002379.

Perret M, Chautems A, Spichiger R, Peixoto M, Savolainen V. 2001. Nectar sugar composition in relation to pollination syndromes in Sinningieae (Gesneriaceae). Ann Bot-London $87: 267-273$. 
1156 Piechowski D, Dötterl S, Gottsberger G. 2010. Pollination biology and floral scent chemistry of

1157

1158

1159

1160

1161

1162

1163

1164

1165

1166

1167

1168

1169

1170

1171

1172

1173

1174

1175

1176

1177

1178

1179

1180

1181

1182

1183

1184

1185

1186

1187

1188

1189

1190

1191

1192

1193

1194 the Neotropical chieropterophilous Parkia pendula. Plant Biol 12:172-182.

Pickrell JK, Marioni JC, Pai AA, Degner JF, Engelhardt BE, Nkadori E, Veyrieras JB, Stephens M, Gilad Y, Pritchard JK. 2010. Understanding mechanisms underlying human gene expression variation with RNA sequencing. Nature 464:768.

Pollastri S, Tattini M. 2011. Flavonols: old compounds for old roles. Ann Bot-London 108:12251233.

Prasad A, Kumar SS, Dessimoz C, Bleuler S, Laule O, Hruz T, Gruissem W, Zimmerman P. 2013. Global regulatory architecture of human, mouse and rat tissue transcriptomes. BMC Genomics 14:716.

Preston JC, Hileman LC, Cubas P. 2011. Reduce, reuse, and recycle: developmental evolution of trait diversification. Am J Bot 98:397-403.

Preston JC, Martinez CC. Hileman LC. 2011. Gradual disintegration of the floral symmetry gene network is implicated in the evolution of a wind-pollination syndrome. P Natl Acad Sci USA 108:2343-2348.

Price MN, Dehal PS, Arkin AP. 2010. FastTree 2-approximately maximum-likelihood trees for large alignments. PLOS One 5:e9490.

Provenzano S, Spelt C, Hosokawa S, Nakamura N, Brugliera F, Demelis L, Geerke DP, Schubert A, Tanaka Y, Quattrocchio F, Koes R. 2014. Genetic control and evolution of anthocyanin methylation. Plant Physiol 165:962-977.

Quattrocchio F, Wing J, van der Woude K, Souer E, de Vetten N, Mol J, Koes R. 1999. Molecular analysis of the anthocyanin 2 gene of petunia and its role in the evolution of flower color. Plant Cell 11:1433-1444.

Raherison ESM, Giguère I, Caron S, Lamara M, MacKay JJ. 2015. Modular organization of the white spruce (Picea glauca) transcriptome reveals functional organization and evolutionary signatures. New Phytol 207:172-187.

Ramírez-Aguirre E, Martén-Rodríguez S, Quesada-Avila G, Quesada M, Martínez-Díaz Y, Oyama K, Espinosa-García FJ. 2019. Reproductive isolation among three sympatric Achimenes species: pre- and post-pollination components. Am J Bot 106:1021-1031.

Ramírez Roa MA. 1987. Revision de Achimenes (Gesneriaceae). PhD dissertation, Universidad Nacional Autonoma de Mexico, Mexico City, Mexico.

Ravasz E, Somera AL, Mongru DA, Oltvai ZN, Barbási AL. 2002. Hierarchical organization of modularity in metabolic networks. Science 297:1551-1555.

Rice-Evans CA, Miller N, Papanga G. 1996. Structure-antioxidant relationships of flavonoids and phenolic acids. Free Radical Bio Med 20:933-956.

Ritchie W, Rajasekhar M, Flamant S, Rasko JE. 2009. Conserved expression patterns predict microRNA targets. PLOS Comput Biol 5:e1000513.

Roalson EH, Roberts WR. 2016. Distinct processes drive diversification in different clades of Gesneriaceae. Syst Biol 65:662-684. 
1195 Roalson EH, Skog LE, Zimmer EA. 2003. Phylogenetic relationships and the diversification of

1196

1197

1198

1199

1200

1201

1202

1203

1204

1205

1206

1207

1208

1209

1210

1211

1212

1213

1214

1215

1216

1217

1218

1219

1220

1221

1222

1223

1224

1225

1226

1227

1228

1229

1230

1231

1232

1233 floral form in Achimenes (Gesneriaceae). Syst Bot 28:593-608.

Roberts WR, Roalson EH, 2017. Comparative transcriptome analyses of flower development in four species of Achimenes (Gesneriaceae). BMC Genomics 18:240.

Roberts WR, Roalson EH. 2018. Phylogenomic analyses reveal extensive gene flow within the magic flowers (Achimenes). Am J Bot 105:726-740.

Romero IG, Ruvinsky I, Gilad Y. 2012. Comparative studies of gene expression and the evolution of gene regulation. Nat Rev Genet 13:505-516.

Ronen G, Carmel-Goren L, Zamir D, Hirschberg J. 2000. An alternative pathway to $\beta$-carotene formation in plant chromoplasts discovered by map-based cloning of Beta and old-gold color mutations in tomato. P Natl Acad Sci USA 97:11102-11107.

Rosas-Guerrero V, Aguilar R, Martén-Rodriguez S, Ashworth L, Lopezaraiza-Mikel M, Bastida JM, Quesada M. 2014. A quantitative review of pollination syndromes: do floral traits predict effective pollinators? Ecol Lett 17:388-400.

Rosas-Saavedra C, Stange C. 2016. Biosynthesis of carotenoids in plants: Enzymes and color. In: Stange C, editor. Carotenoids in nature: biosynthesis, regulation and function. Vol. 79. Springer. p. 35-69.

Saito K, Yamazaki M. 2002. Biochemistry and molecular biology of the late-stage of biosynthesis of anthocyanin: lessons from Perilla frutescens as a model plant. New Phytol 155:9-23.

Sakata Y, Aoki N, Tsunematsu S, Nishikouri H, Johjima T. 1995. Petal coloration and pigmentation of tree peony bred and selected in Daikon island (Shimane Prefecture). J Jpn Soc Hortic Sci 65:351-357.

Sauquet H, Magallón S. 2018. Key questions and challenges in angiosperm macroevolution. New Phytol 219:1170-1187.

Scoville AG, Barnett LL, Bodbyl-Roels S, Kelly JK, Hileman LC. 2011. Differential regulation of a MYB transcription factor is correlated with transgenerational epigenetic inheritance of trichome density in Mimulus guttatus. New Phytol 191:251-263.

Serrano-Serrano ML, Marcionetti A, Perret M, Salamin N. 2017a. Transcriptome resources for an endemic Neotropical plant lineage (Gesneriaceae). Appl Plant Sci 5:1600135.

Serrano-Serrano ML, Rolland J, Clark JL, Salamin N, Perret M. 2017b. Hummingbird pollination and the diversification of angiosperms: an old and successful association in Gesneriaceae. P Roy Soc B-Biol Sci 284:20162816.

Shahan R, Zawora C, Wight H, Sittmann J, Wang W, Mount SM, Liu Z. 2018. Consensus coexpression network analysis identifies key regulators of flower and fruit development in wild strawberry. Plant Physiol 178:202-216.

Simão FA, Waterhouse RM, Ioannidis P, Kriventseva EV, Zbodnov EM. 2015. BUSCO: assessing genome assembly and annotation completeness with single-copy orthologs. Bioinformatics 31:3210-3212. 
1234

1235

1236

1237

1238

1239

1240

1241

1242

1243

1244

1245

1246

1247

1248

1249

1250

1251

1252

1253

1254

1255

1256

1257

1258

1259

1260

1261

1262

1263

1264

1265

1266

1267

1268

1269

1270

1271

Sletvold N, Grindeland JM, Ågren J. 2010. Pollinator-mediated selection on floral display, spur length and flowering phenology in the deceptive orchid Dactylorhiza lapponica. New Phytol 188:385-392.

Sletvold N, Trunschke J, Smit M, Verbeek J, Ågren J. 2016. Strong pollinator-mediated selection for increased flower brightness and contrast in a deceptive orchid. Evolution 70:716-724.

Smith SA, Dunn CW. 2008. Phyutility: a phyloinformatics tool for trees, alignments and molecular data. Bioinformatics 24:715-716.

Smith SD, Rausher MD. 2011. Gene loss and parallel evolution contribute to species difference in flower color. Mol Biol Evol 28:2799-2810.

Smith SD, Wang S, Rausher MD. 2013. Functional evolution of an anthocyanin pathway enzyme during a flower color transition. Mol Biol Evol 30:602-612.

Song L, Langfelder P, Horvath S. 2012. Comparison of co-expression measures: mutual information, correlation, and model based indices. BMC Bioinformatics 13:328.

Springob K. Nakajima JI, Yamazaki M, Saito K. 2003. Recent advances in the biosynthesis and accumulation of anthocyanins. Nat Prod Rep 20:288-303.

Stamatakis A. 2014. RAxML version 8: a tool for phylogenetic analysis and post-analysis of large phylogenies. Bioinformatics 30:1312-1313.

Stebbins GL. 1970. Adaptive radiation of reproductive characteristics in angiosperms, I: pollination mechanisms. Annu Rev Ecol Syst 1:307-326.

Sun B, Xu Y, Ng KH, Ito T. 2009. A timing mechanism for stem cell maintenance and differentiation in the Arabidopsis floral meristem. Gene Dev 23:1791-1804.

Supek F, Bošnjak M, Škunca N, Šmuc T. 2011. REVIGO summarizes and visualizes long lists of gene ontology terms. PLOS One 6:e21800.

Suyama M, Torrents D, Bork P. 2006. PAL2NAL: robust conversion of protein sequence alignments into the corresponding codon alignments. Nucleic Acids Res 34:W609-W612.

Suzuki JY, Amore TD, Calla B, Palmer NA, Scully ED, Sattler SE, Sarath G, Lichty JS, Myers RY, Keith LM, Matsumoto TK. 2017. Organ-specific transcriptome profiling of metabolic and pigment biosynthetic pathways in the floral ornamental progenitor species Anthurium amnicola Dressler. Sci Rep-UK 7:1596.

Tahara S. 2007. A journey of twenty-five years through the ecological biochemistry of flavonoids. Biosci Biotechnol Biochem 71:1387-1404.

Tanaka Y, Sasaki N, Ohmiya A. 2008. Biosynthesis of plant pigments: anthocyanins, betalains and carotenoids. Plant J 54:733-749.

Tang H, Wang X, Bowers JE, Ming R, Alam M, Paterson AH. 2008. Unraveling ancient hexaploidy through multiply-aligned angiosperm gene maps. Genome Res 18:1944-1954.

Tröbner W, Ramirez L, Motte P, Hue I, Huijser P, Löning WE, Saedler H, Sommer H, SchwarzSommer Z. 1992. GLOBOSA: a homeotic gene which interacts with DEFICIENS in the control of Antirrhinum floral organogenesis. EMBO J 11:4693-4704. 
1272 Uebbing S, Künster A, Mäkinen H, Backström N, Bolivar P, Burri R, Dutoit L, Mugal CF, Nater

1273

1274

1275

1276

1277

1278

1279

1280

1281

1282

1283

1284

1285

1286

1287

1288

1289

1290

1291

1292

1293

1294

1295

1296

1297

1298

1299

1300

1301

1302

1303

1304

1305

1306

1307

1308

1309

1310

1311

A, Aken B, Flicek P. 2016. Divergence in gene expression within and between two closely related flycatcher species. Mol Ecol 25:2015-2028.

The UniProt Consortium. 2017. UniProt: the universal protein knowledgebase. Nucleic Acids Res 45:D158-D169.

Van Dam S, Võsa U, van der Graaf A, Franke L, de Magalhaes JP. 2018. Gene co-expression analysis for functional classification and gene-disease predictions. Brief Bioinform 19:575-592.

Vandelook F, Janssens SB, Gijbels P, Fischer E, Van den Ende W, Honnay O, Abrahamczyk S. 2019. Nectar traits differ between pollination syndromes in Balsaminaceae. Ann BotLondon 124:269-279.

Van der Niet T, Johnson SD. 2012. Phylogenetic evidence for pollinator-driven diversification of angiosperms. Trends Ecol Evol 27: 353-361.

Vincent CA, Enrico SC. 2004. A temporal and morphological framework for flower development in Antirrhinum majus. Can J Botany 82:681-690.

Waites R, Selvadurai HR, Oliver IR, Hudson A. 1998. The PHANTASTICA gene encodes a MYB transcription factor involved in growth and dorsoventrality of lateral organs in Antirrhinum. Cell 93:779-789.

Wang M, Wang G, Ji J, Wang J. 2009. The effect of pds gene silencing on chloroplast pigment composition, thylakoid membrane structure and photosynthesis efficiency in tobacco plants. Plant Sci 177:222-226.

Wessinger CA, Rausher MD. 2014. Ecological transition predictably associated with gene degeneration. Mol Biol Evol 32:347-354.

West-Eberhard MJ. 1989. Phenotypic plasticity and the origins of diversity. Ann Rev Ecol Syst 20:249-278.

Whitney HM, Glover BJ, Walker R, Ellis AG. 2011. The contribution of epidermal structure to flower colour in the South African flora. Curtis's Botanical Magazine 28:349-371.

Wiehler H. 1983. A synopsis of the neotropical Gesneriaceae. Selbyana 6:1-219.

Winkel-Shirley B. 2002. Biosynthesis of flavonoids and effects of stress. Curr Opinion Plant Biol 5:218-223.

Wu CA, Streisfeld MA, Nutter LI, Cross KA. 2013. The genetic basis of a rare flower color polymorphism in Mimulus lewisii provides insight into the repeatability of evolution. PLOS One 8:12.

Yang Y, Smith SA. 2014. Orthology inference in nonmodel organisms using transcriptomes and low-coverage genomes: improving accuracy and matrix occupancy for phylogenomics. Mol Biol Evol 31:3081-3092.

Yang Z. 2007. PAML 4: phylogenetic analysis by maximum likelihood. Mol Biol Evol 24:15861591.

Yant L, Collani S, Puzey J, Levy C, Kramer EM. 2015. Molecular basis for three-dimensional elaboration of the Aquilegia petal spur. P Roy Soc B-Biol Sci 282:20142778.

Peer) reviewing PDF | (2019:10:42302:1:1:NEW 10 Feb 2020) 
1312 Yuan YW, Rebocho AB, Sagawa JM, Stanley LE, Bradshaw HD. 2016. Competition between

1313

1314

1315

1316

1317

1318

1319

1320

1321

1322

1323

1324

1325

1326

1327

1328 anthocyanin and flavonol biosynthesis produces spatial pattern variation of floral pigments between Mimulus species. P Natl Acad Sci USA 113: 2448-2453.

Yuan YW, Sagawa JM, Young RC, Christensen BJ, Bradshaw HD. 2013. Genetic dissection of a major anthocyanin QTL contributing to pollinator-mediated reproductive isolation between sister species of Mimulus. Genetics 194:255-263.

Zhang JS, Li Z, Zhao J, Zhang S, Quan H, Zhao H, He C. 2014. Deciphering the Physalis floridana double-layered-lantern1 mutant provides insights into the functional divergence of the GLOBOSA duplicates within the Solanaceae. Plant Physiol 164:748-764.

Zhang W, Kramer EM, Davis CC. 2010. Floral symmetry genes and the origin and maintenance of zygomorphy in a plant-pollinator mutualism. P Natl Acad Sci USA 107:6388-6393.

Zhou XR, Wang YZ, Smith JF, Chen R. 2008. Altered expression patterns of TCP and MYB genes relating to the floral developmental transition from initial zygomorphy to actinomorphy in Bournea (Gesneriaceae). New Phytol 178:532-543.

Zuo J, Niu Q-W, Frugis G, Chua N-H. 2002. The WUSCHEL gene promotes vegetative-toembryonic transition in Arabidopsis. Plant J 30:349-359. 
Table $\mathbf{1}$ (on next page)

Definition of pollination syndromes in Achimenes, Eucodonia, and Gesneria 
1 Table 1. Definition of pollination syndromes in Achimenes, Eucodonia, and Gesneria.

2

\begin{tabular}{|c|c|c|c|c|}
\hline Pollination syndrome & Primary flower color & Flower shape & $\begin{array}{l}\text { Presence of } \\
\text { corolla spurs }\end{array}$ & Species \\
\hline \multirow[t]{3}{*}{ Melittophily (bees) } & \multirow{3}{*}{$\begin{array}{l}\text { White or Purple with } \\
\text { yellow or purple } \\
\text { spotted throat }\end{array}$} & \multirow[t]{3}{*}{ Funnelform } & \multirow[t]{3}{*}{ No } & Achimenes candida \\
\hline & & & & Achimenes misera \\
\hline & & & & Eucodonia verticillata \\
\hline \multirow{5}{*}{$\begin{array}{l}\text { Ornithophily } \\
\text { (hummingbirds) }\end{array}$} & \multirow[t]{5}{*}{ Red and yellow } & \multirow{5}{*}{$\begin{array}{l}\text { Tubular, } \\
\text { Salverform }\end{array}$} & \multirow[t]{5}{*}{ No } & Achimenes admirabilis \\
\hline & & & & Achimenes antirrhina \\
\hline & & & & Achimenes erecta \\
\hline & & & & Achimenes pedunculata \\
\hline & & & & Gesneria cuneifolia \\
\hline \multirow{4}{*}{$\begin{array}{l}\text { Psychophily } \\
\text { (butterflies) }\end{array}$} & \multirow[t]{4}{*}{ Purple } & \multirow[t]{4}{*}{ Salverform } & \multirow[t]{4}{*}{ Yes } & Achimenes cettoana \\
\hline & & & & Achimenes grandiflora \\
\hline & & & & Achimenes longiflora \\
\hline & & & & Achimenes patens \\
\hline
\end{tabular}


Table 2 (on next page)

Gene Ontology enrichment for the network hubs 
1 Table 2. Gene Ontology enrichment for the network hubs.

\begin{tabular}{|c|c|c|c|c|c|c|}
\hline & GO ID & Term & Annotated & Significant & Expected & $\mathrm{P}$ \\
\hline \multirow{24}{*}{ A. Bud stage Hubs } & GO:0031325 & positive regulation of cellular metabolic process & 224 & 16 & 14.52 & 0.000 \\
\hline & GO:0006607 & NLS-bearing protein import into nucleus & 7 & 4 & 0.45 & 0.001 \\
\hline & GO:0015991 & ATP hydrolysis coupled proton transport & 9 & 4 & 0.58 & 0.002 \\
\hline & GO:0046470 & phosphatidylcholine metabolic process & 9 & 4 & 0.58 & 0.002 \\
\hline & GO:0018298 & protein-chromophore linkage & 32 & 7 & 2.07 & 0.004 \\
\hline & GO:0070507 & regulation of microtubule cytoskeleton organization & 6 & 3 & 0.39 & 0.005 \\
\hline & GO: 1990778 & protein localization to cell periphery & 6 & 3 & 0.39 & 0.005 \\
\hline & GO:0051592 & response to calcium ion & 7 & 3 & 0.45 & 0.008 \\
\hline & GO:0006412 & translation & 233 & 24 & 15.10 & 0.009 \\
\hline & GO:0042594 & response to starvation & 60 & 10 & 3.89 & 0.012 \\
\hline & GO:0007035 & vacuolar acidification & 8 & 3 & 0.52 & 0.012 \\
\hline & GO:0006534 & cysteine metabolic process & 9 & 4 & 0.58 & 0.012 \\
\hline & GO:0009833 & plant-type primary cell wall biogenesis & 15 & 4 & 0.97 & 0.013 \\
\hline & GO:0048831 & regulation of shoot system development & 123 & 12 & 7.97 & 0.017 \\
\hline & GO:0032509 & endosome transport via multivesicular body sorting pathway & 9 & 3 & 0.58 & 0.017 \\
\hline & GO:0051568 & histone H3-K4 methylation & 9 & 3 & 0.58 & 0.017 \\
\hline & GO:0000271 & polysaccharide biosynthetic process & 123 & 11 & 7.97 & 0.023 \\
\hline & GO:0006732 & coenzyme metabolic process & 116 & 8 & 7.52 & 0.023 \\
\hline & GO:0010338 & leaf formation & 5 & 2 & 0.32 & 0.037 \\
\hline & GO:0048564 & photosystem I assembly & 5 & 2 & 0.32 & 0.037 \\
\hline & GO:0017001 & antibiotic catabolic process & 26 & 3 & 1.69 & 0.037 \\
\hline & GO:0044282 & small molecule catabolic process & 75 & 10 & 4.86 & 0.038 \\
\hline & GO:0006833 & water transport & 13 & 3 & 0.84 & 0.048 \\
\hline & GO ID & Term & Annotated & Significant & Expected & $\mathrm{P}$ \\
\hline \multirow[t]{17}{*}{ B. D stage Hubs } & GO:0006412 & translation & 233 & 30 & 16.03 & 0.001 \\
\hline & GO:0042939 & tripeptide transport & 8 & 4 & 0.55 & 0.001 \\
\hline & GO:0016117 & carotenoid biosynthetic process & 20 & 6 & 1.38 & 0.002 \\
\hline & GO:0006744 & ubiquinone biosynthetic process & 5 & 3 & 0.34 & 0.003 \\
\hline & GO:0032259 & methylation & 88 & 15 & 6.05 & 0.004 \\
\hline & GO:0045814 & negative regulation of gene expression, epigenetic & 37 & 5 & 2.55 & 0.005 \\
\hline & GO:0009206 & purine ribonucleoside triphosphate biosynthetic process & 44 & 6 & 3.03 & 0.013 \\
\hline & GO:0000271 & polysaccharide biosynthetic process & 123 & 11 & 8.46 & 0.013 \\
\hline & GO:0009873 & ethylene-activated signaling pathway & 78 & 12 & 5.37 & 0.014 \\
\hline & GO:0015991 & ATP hydrolysis coupled proton transport & 9 & 3 & 0.62 & 0.020 \\
\hline & GO:0009718 & anthocyanin-containing compound biosynthetic process & 26 & 5 & 1.79 & 0.030 \\
\hline & GO:0000018 & regulation of DNA recombination & 5 & 2 & 0.34 & 0.041 \\
\hline & GO:0015976 & carbon utilization & 5 & 2 & 0.34 & 0.041 \\
\hline & GO:0034314 & Arp2/3 complex-mediated actin nucleation & 5 & 2 & 0.34 & 0.041 \\
\hline & GO:0080144 & amino acid homeostasis & 5 & 2 & 0.34 & 0.041 \\
\hline & GO:1901615 & organic hydroxy compound metabolic process & 121 & 9 & 8.32 & 0.041 \\
\hline & GO:0000902 & cell morphogenesis & 174 & 9 & 11.97 & 0.042 \\
\hline
\end{tabular}


Table 3 (on next page)

Gene Ontology enrichment for the network periphery 
1 Table 3. Gene Ontology enrichment for the network periphery.

\begin{tabular}{|c|c|c|c|c|c|c|}
\hline & GO.ID & Term & Annotated & Significant & Expected & $\mathrm{P}$ \\
\hline \multirow[t]{24}{*}{ A. Bud stage periphery } & GO:0016579 & protein deubiquitination & 24 & 16 & 8.70 & 0.002 \\
\hline & GO:0048825 & cotyledon development & 21 & 12 & 7.61 & 0.005 \\
\hline & GO:0015846 & polyamine transport & 5 & 5 & 1.81 & 0.006 \\
\hline & GO:0034314 & Arp2/3 complex-mediated actin nucleation & 5 & 5 & 1.81 & 0.006 \\
\hline & GO:0006020 & inositol metabolic process & 12 & 7 & 4.35 & 0.011 \\
\hline & GO:0010229 & inflorescence development & 9 & 7 & 3.26 & 0.014 \\
\hline & GO:0009744 & response to sucrose & 31 & 16 & 11.24 & 0.021 \\
\hline & GO:1903338 & regulation of cell wall organization or biogenesis & 11 & 7 & 3.99 & 0.026 \\
\hline & GO:0006809 & nitric oxide biosynthetic process & 6 & 5 & 2.18 & 0.026 \\
\hline & GO:0009773 & photosynthetic electron transport in photosystem I & 8 & 6 & 2.90 & 0.030 \\
\hline & GO:0034613 & cellular protein localization & 183 & 75 & 66.34 & 0.031 \\
\hline & GO:0006004 & fucose metabolic process & 12 & 8 & 4.35 & 0.032 \\
\hline & GO:0009926 & auxin polar transport & 42 & 22 & 15.23 & 0.037 \\
\hline & GO:0010090 & trichome morphogenesis & 38 & 19 & 13.78 & 0.041 \\
\hline & GO:0009414 & response to water deprivation & 182 & 69 & 65.98 & 0.045 \\
\hline & GO:0009873 & ethylene-activated signaling pathway & 78 & 38 & 28.28 & 0.046 \\
\hline & GO:0007166 & cell surface receptor signaling pathway & 93 & 42 & 33.71 & 0.046 \\
\hline & GO:0006163 & purine nucleotide metabolic process & 96 & 38 & 34.80 & 0.047 \\
\hline & GO:0035195 & gene silencing by miRNA & 15 & 7 & 5.44 & 0.048 \\
\hline & GO:0016236 & macroautophagy & 21 & 9 & 7.61 & 0.048 \\
\hline & GO:0055083 & monovalent inorganic anion homeostasis & 13 & 5 & 4.71 & 0.048 \\
\hline & GO:0051338 & regulation of transferase activity & 38 & 13 & 13.78 & 0.048 \\
\hline & GO:0006473 & protein acetylation & 23 & 7 & 8.34 & 0.048 \\
\hline & GO.ID & Term & Annotated & Significant & Expected & $\mathrm{P}$ \\
\hline \multirow[t]{17}{*}{ B. D stage periphery } & GO:0045490 & pectin catabolic process & 20 & 19 & 12.88 & 0.002 \\
\hline & GO:0007166 & cell surface receptor signaling pathway & 93 & 71 & 59.90 & 0.006 \\
\hline & GO:0000398 & mRNA splicing, via spliceosome & 46 & 38 & 29.63 & 0.007 \\
\hline & GO:0009740 & gibberellic acid mediated signaling pathway & 31 & 24 & 19.97 & 0.015 \\
\hline & GO:0006310 & DNA recombination & 54 & 41 & 34.78 & 0.015 \\
\hline & GO:0009909 & regulation of flower development & 80 & 57 & 51.53 & 0.017 \\
\hline & GO:0009058 & biosynthetic process & 2121 & 1360 & 1366.19 & 0.019 \\
\hline & GO:0042545 & cell wall modification & 34 & 26 & 21.90 & 0.021 \\
\hline & GO:0001708 & cell fate specification & 13 & 12 & 8.37 & 0.027 \\
\hline & GO:0042538 & hyperosmotic salinity response & 25 & 21 & 16.10 & 0.027 \\
\hline & GO:0002181 & cytoplasmic translation & 21 & 18 & 13.53 & 0.029 \\
\hline & GO:0006897 & endocytosis & 40 & 27 & 25.76 & 0.029 \\
\hline & GO:0010197 & polar nucleus fusion & 20 & 17 & 12.88 & 0.039 \\
\hline & GO:0071702 & organic substance transport & 492 & 319 & 316.91 & 0.046 \\
\hline & GO:0006949 & syncytium formation & 7 & 7 & 4.51 & 0.046 \\
\hline & GO:0008295 & spermidine biosynthetic process & 7 & 7 & 4.51 & 0.046 \\
\hline & GO:0009963 & positive regulation of flavonoid biosynthetic process & 7 & 7 & 4.51 & 0.046 \\
\hline
\end{tabular}


Table 4 (on next page)

Gene Ontology enrichment in Bud stage modules correlated to pollination syndrome 
Table 4. Gene Ontology enrichment in Bud stage modules correlated to pollination syndrome.

\begin{tabular}{|c|c|c|c|c|c|}
\hline \multicolumn{6}{|c|}{ A. Bee pollination } \\
\hline GO.ID & Term & Annotated & Significant & Expected & $\mathrm{P}$ \\
\hline GO:0046686 & response to cadmium ion & 99 & 16 & 5.69 & 0.000 \\
\hline GO:0010104 & regulation of ethylene-activated signaling pathway & 12 & 3 & 0.69 & 0.003 \\
\hline GO:0010189 & vitamin $\mathrm{E}$ biosynthetic process & 8 & 3 & 0.46 & 0.008 \\
\hline GO:0070085 & glycosylation & 72 & 7 & 4.13 & 0.009 \\
\hline GO:0009226 & nucleotide-sugar biosynthetic process & 21 & 5 & 1.21 & 0.011 \\
\hline GO:0042136 & neurotransmitter biosynthetic process & 9 & 4 & 0.52 & 0.018 \\
\hline GO:0070592 & cell wall polysaccharide biosynthetic process & 32 & 4 & 1.84 & 0.018 \\
\hline GO:0051173 & positive regulation of nitrogen compound metabolic process & 192 & 4 & 11.03 & 0.019 \\
\hline GO:0046777 & protein autophosphorylation & 71 & 9 & 4.08 & 0.019 \\
\hline GO:0009651 & response to salt stress & 215 & 19 & 12.35 & 0.022 \\
\hline GO:0009738 & abscisic acid-activated signaling pathway & 135 & 11 & 7.75 & 0.028 \\
\hline GO:0015718 & monocarboxylic acid transport & 12 & 3 & 0.69 & 0.028 \\
\hline GO:0046889 & positive regulation of lipid biosynthetic process & 12 & 3 & 0.69 & 0.029 \\
\hline GO:0006809 & nitric oxide biosynthetic process & 5 & 2 & 0.29 & 0.029 \\
\hline GO:0006974 & cellular response to DNA damage stimulus & 99 & 9 & 5.69 & 0.035 \\
\hline GO:0010099 & regulation of photomorphogenesis & 6 & 2 & 0.34 & 0.042 \\
\hline GO:0071805 & potassium ion transmembrane transport & 6 & 2 & 0.34 & 0.042 \\
\hline \multicolumn{6}{|c|}{ B. Butterfly pollination } \\
\hline GO.ID & Term & Annotated & Significant & Expected & $\mathrm{P}$ \\
\hline GO:0009415 & response to water & 155 & 13 & 11.47 & 0.016 \\
\hline GO:0008380 & RNA splicing & 122 & 16 & 9.03 & 0.016 \\
\hline GO:0010223 & secondary shoot formation & 14 & 4 & 1.04 & 0.016 \\
\hline GO:0009624 & response to nematode & 15 & 4 & 1.11 & 0.021 \\
\hline GO:0009695 & jasmonic acid biosynthetic process & 9 & 3 & 0.67 & 0.024 \\
\hline GO:0090691 & formation of plant organ boundary & 9 & 3 & 0.67 & 0.024 \\
\hline
\end{tabular}




\begin{tabular}{|c|c|c|c|c|c|}
\hline GO:0006950 & response to stress & 1173 & 83 & 86.79 & 0.028 \\
\hline GO:0006353 & DNA-templated transcription, termination & 17 & 4 & 1.26 & 0.032 \\
\hline GO:0009567 & double fertilization forming a zygote and endosperm & 11 & 3 & 0.81 & 0.042 \\
\hline GO:0009835 & fruit ripening & 11 & 3 & 0.81 & 0.042 \\
\hline GO:0009607 & response to biotic stimulus & 374 & 35 & 27.67 & 0.045 \\
\hline GO:0010216 & maintenance of DNA methylation & 5 & 2 & 0.37 & 0.047 \\
\hline GO:0010363 & regulation of plant-type hypersensitive response & 5 & 2 & 0.37 & 0.047 \\
\hline GO:0071577 & zinc ion transmembrane transport & 5 & 2 & 0.37 & 0.047 \\
\hline GO:0009825 & multidimensional cell growth & 19 & 4 & 1.41 & 0.047 \\
\hline \multicolumn{6}{|c|}{ C. Hummingbird pollination } \\
\hline GO.ID & Term & Annotated & Significant & Expected & $\mathrm{P}$ \\
\hline GO:0009451 & RNA modification & 353 & 89 & 62.34 & 0.000 \\
\hline GO:1900618 & regulation of shoot system morphogenesis & 9 & 5 & 1.59 & 0.001 \\
\hline GO:0009826 & unidimensional cell growth & 95 & 24 & 16.78 & 0.002 \\
\hline GO:0016556 & mRNA modification & 35 & 11 & 6.18 & 0.002 \\
\hline GO:0006012 & galactose metabolic process & 5 & 4 & 0.88 & 0.004 \\
\hline GO:0031425 & chloroplast RNA processing & 18 & 8 & 3.18 & 0.008 \\
\hline GO:0008643 & carbohydrate transport & 44 & 15 & 7.77 & 0.010 \\
\hline GO:1905428 & regulation of plant organ formation & 6 & 4 & 1.06 & 0.011 \\
\hline GO:0006888 & ER to Golgi vesicle-mediated transport & 19 & 8 & 3.36 & 0.011 \\
\hline GO:0044272 & sulfur compound biosynthetic process & 58 & 12 & 10.24 & 0.011 \\
\hline GO:0045944 & positive regulation of transcription by RNA polymerase II & 37 & 13 & 6.53 & 0.013 \\
\hline GO:0002239 & response to oomycetes & 18 & 6 & 3.18 & 0.019 \\
\hline GO:0006813 & potassium ion transport & 16 & 7 & 2.83 & 0.020 \\
\hline GO:0043650 & dicarboxylic acid biosynthetic process & 10 & 5 & 1.77 & 0.020 \\
\hline GO:0051053 & negative regulation of DNA metabolic process & 7 & 4 & 1.24 & 0.022 \\
\hline GO:0051301 & cell division & 147 & 30 & 25.96 & 0.027 \\
\hline GO:0000079 & regulation of cyclin-dependent protein serine/threonine kinase activity & 11 & 5 & 1.94 & 0.031 \\
\hline GO:0046131 & pyrimidine ribonucleoside metabolic process & 12 & 3 & 2.12 & 0.031 \\
\hline
\end{tabular}


GO:0032509 endosome transport via multivesicular body sorting pathway 


\section{Table 5 (on next page)}

Gene Ontology enrichment in D stage modules correlated to pollination syndrome 
Table 5. Gene Ontology enrichment in D stage modules correlated to pollination syndrome.

\begin{tabular}{|c|c|c|c|c|c|}
\hline \multicolumn{6}{|c|}{ A. Bee pollination } \\
\hline GO.ID & Term & Annotated & Significant & Expected & $\mathrm{P}$ \\
\hline GO:0061014 & positive regulation of mRNA catabolic process & 6 & 3 & 0.26 & 0.002 \\
\hline GO:0044093 & positive regulation of molecular function & 46 & 4 & 2.01 & 0.006 \\
\hline GO:0046686 & response to cadmium ion & 99 & 10 & 4.33 & 0.011 \\
\hline GO:0006974 & cellular response to DNA damage stimulus & 99 & 8 & 4.33 & 0.017 \\
\hline GO:0009395 & phospholipid catabolic process & 5 & 2 & 0.22 & 0.017 \\
\hline GO:0009969 & xyloglucan biosynthetic process & 5 & 2 & 0.22 & 0.017 \\
\hline GO:0006544 & glycine metabolic process & 7 & 2 & 0.31 & 0.035 \\
\hline GO:0043488 & regulation of mRNA stability & 7 & 2 & 0.31 & 0.035 \\
\hline GO:0016569 & covalent chromatin modification & 58 & 4 & 2.54 & 0.044 \\
\hline GO.ID & Term & Annotated & Significant & Expected & $\mathrm{P}$ \\
\hline GO:0009853 & photorespiration & 19 & 7 & 2.00 & 0.002 \\
\hline GO:0043622 & cortical microtubule organization & 13 & 5 & 1.37 & 0.008 \\
\hline GO:0031349 & positive regulation of defense response & 51 & 8 & 5.37 & 0.010 \\
\hline GO:0006575 & cellular modified amino acid metabolic process & 35 & 7 & 3.69 & 0.018 \\
\hline GO:0070919 & production of siRNA involved in chromatin silencing by small RNA & 6 & 3 & 0.63 & 0.018 \\
\hline GO:0042398 & cellular modified amino acid biosynthetic process & 7 & 3 & 0.74 & 0.029 \\
\hline GO:0009808 & lignin metabolic process & 22 & 5 & 2.32 & 0.031 \\
\hline GO:2000030 & regulation of response to red or far red light & 9 & 3 & 0.95 & 0.031 \\
\hline GO:0019220 & regulation of phosphate metabolic process & 39 & 6 & 4.11 & 0.031 \\
\hline
\end{tabular}




\begin{tabular}{|c|c|c|c|c|c|}
\hline GO:0051028 & mRNA transport & 44 & 9 & 4.63 & 0.041 \\
\hline GO:0006833 & water transport & 13 & 4 & 1.37 & 0.043 \\
\hline GO:0043902 & positive regulation of multi-organism process & 22 & 4 & 2.32 & 0.044 \\
\hline GO.ID & Term & Annotated & Significant & Expected & \\
\hline GO:0009960 & endosperm development & 10 & 6 & 1.53 & 0.002 \\
\hline GO:0007163 & establishment or maintenance of cell polarity & 5 & 4 & 0.77 & 0.002 \\
\hline GO:0010224 & response to UV-B & 24 & 9 & 3.67 & 0.007 \\
\hline GO:0009089 & lysine biosynthetic process via diaminopimelate & 7 & 4 & 1.07 & 0.013 \\
\hline GO:0009451 & RNA modification & 353 & 70 & 54.01 & 0.015 \\
\hline GO:0007166 & cell surface receptor signaling pathway & 86 & 21 & 13.16 & 0.018 \\
\hline GO:0008285 & negative regulation of cell proliferation & 8 & 4 & 1.22 & 0.023 \\
\hline GO:0033014 & tetrapyrrole biosynthetic process & 39 & 6 & 5.97 & 0.024 \\
\hline GO:0009267 & cellular response to starvation & 47 & 14 & 7.19 & 0.025 \\
\hline GO:0006635 & fatty acid beta-oxidation & 16 & 6 & 2.45 & 0.026 \\
\hline GO:0016556 & mRNA modification & 35 & 9 & 5.36 & 0.026 \\
\hline GO:0048438 & floral whorl development & 69 & 14 & 10.56 & 0.028 \\
\hline GO:0010103 & stomatal complex morphogenesis & 14 & 5 & 2.14 & 0.028 \\
\hline GO:0006383 & transcription by RNA polymerase III & 5 & 3 & 0.77 & 0.028 \\
\hline GO:0040008 & regulation of growth & 126 & 26 & 19.28 & 0.029 \\
\hline GO:0010019 & chloroplast-nucleus signaling pathway & 9 & 4 & 1.38 & 0.036 \\
\hline GO:0010506 & regulation of autophagy & 9 & 4 & 1.38 & 0.036 \\
\hline
\end{tabular}


GO:0031425 chloroplast RNA processing

GO:0051187 cofactor catabolic process

GO:0002238 response to molecule of fungal origin

GO:0032958 inositol phosphate biosynthetic process

GO:0042219 cellular modified amino acid catabolic process

GO:1905428 regulation of plant organ formation

protein-DNA complex assembly

\begin{tabular}{rlll}
18 & 6 & 2.75 & 0.045 \\
34 & 9 & 5.20 & 0.050 \\
6 & 3 & 0.92 & 0.050 \\
6 & 3 & 0.92 & 0.050 \\
6 & 3 & 0.92 & 0.050 \\
6 & 3 & 0.92 & 0.050 \\
45 & 8 & 6.89 & 0.050 \\
\hline
\end{tabular}




\section{Figure 1}

Achimenes flowers and the sampled developmental stages

Flowers of the twelve sampled species: (A) A. admirabilis, (B) A. antirrhina, (C) A. candida, (D) A. cettoana, (E) A. erecta, (F) A. grandiflora, (G) A. longiflora, (H) A. misera, (I) A. patens, (J) A. pedunculata, (K) E. verticillata, and (L) G. cuneifolia. Sampled Bud and D stage

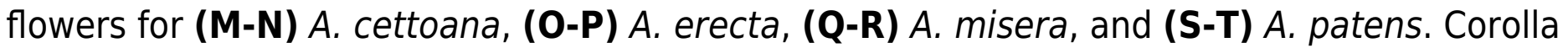
spurs found in (U) A. grandiflora and (V) A. patens are indicated with arrows. Scale bars equal $1 \mathrm{~cm}$. Abbreviations: A, Achimenes; E, Eucudonia; G, Gesneria.
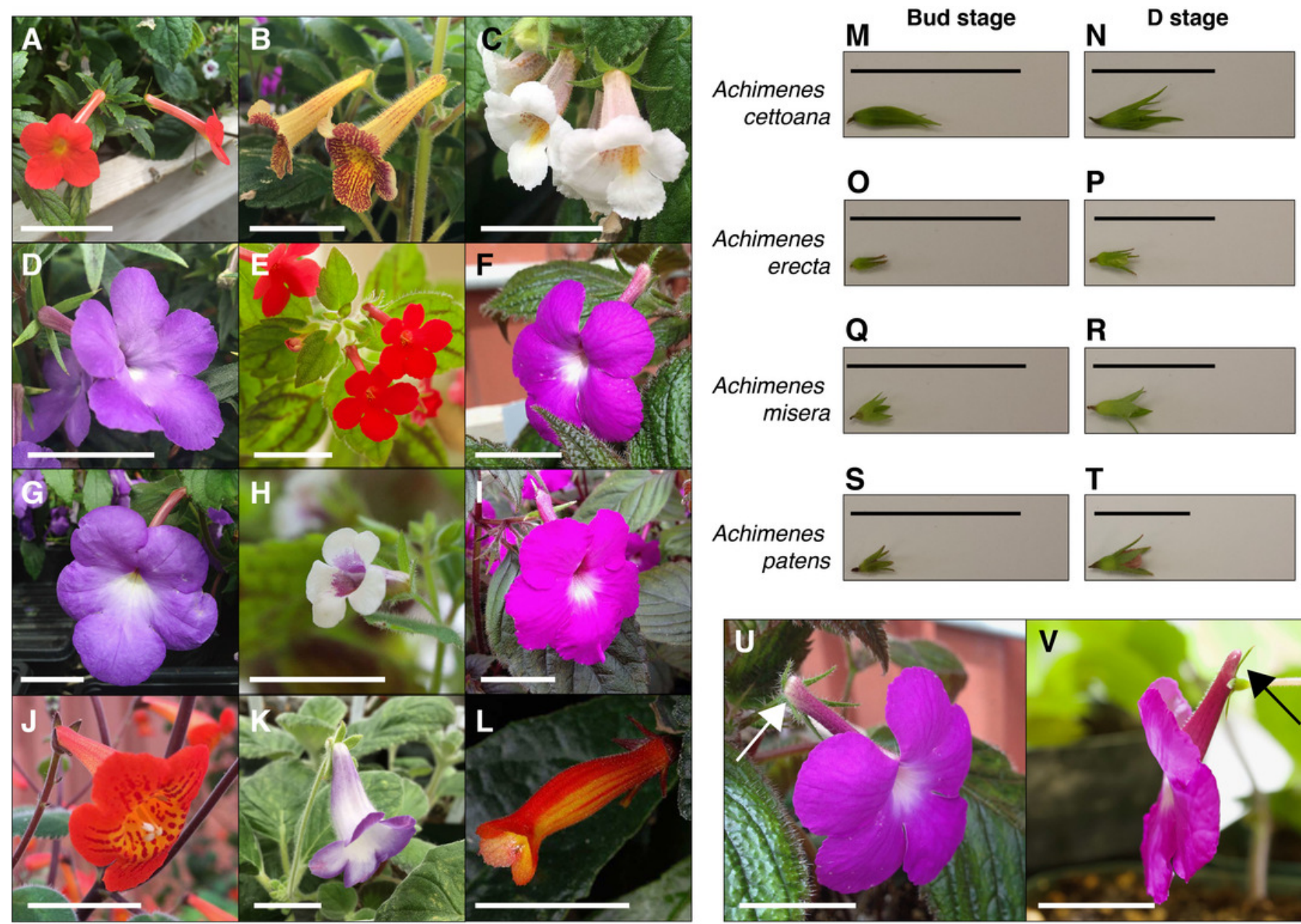

Scale bars equals $1 \mathrm{~cm}$.

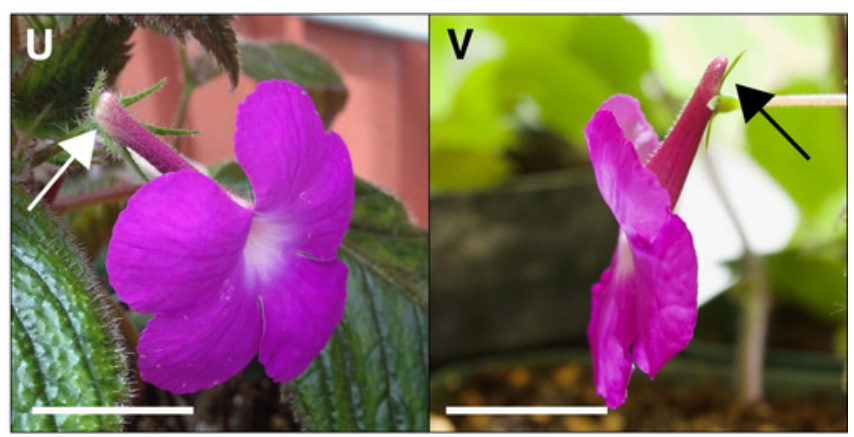


Figure 2

Phylogenetic relationships and floral traits in Achimenes

Phylogeny of Achimenes adapted from Roberts and Roalson (2018). Clade 1 and Clade 2 (sensu Roalson et al. 2003) are indicated on the phylogeny with a circle and box, respectively. Flower traits for each species are shown at the tips of the phylogeny, including flower color, flower shape, presence of corolla spurs, pollination syndrome, and pollinator observations.

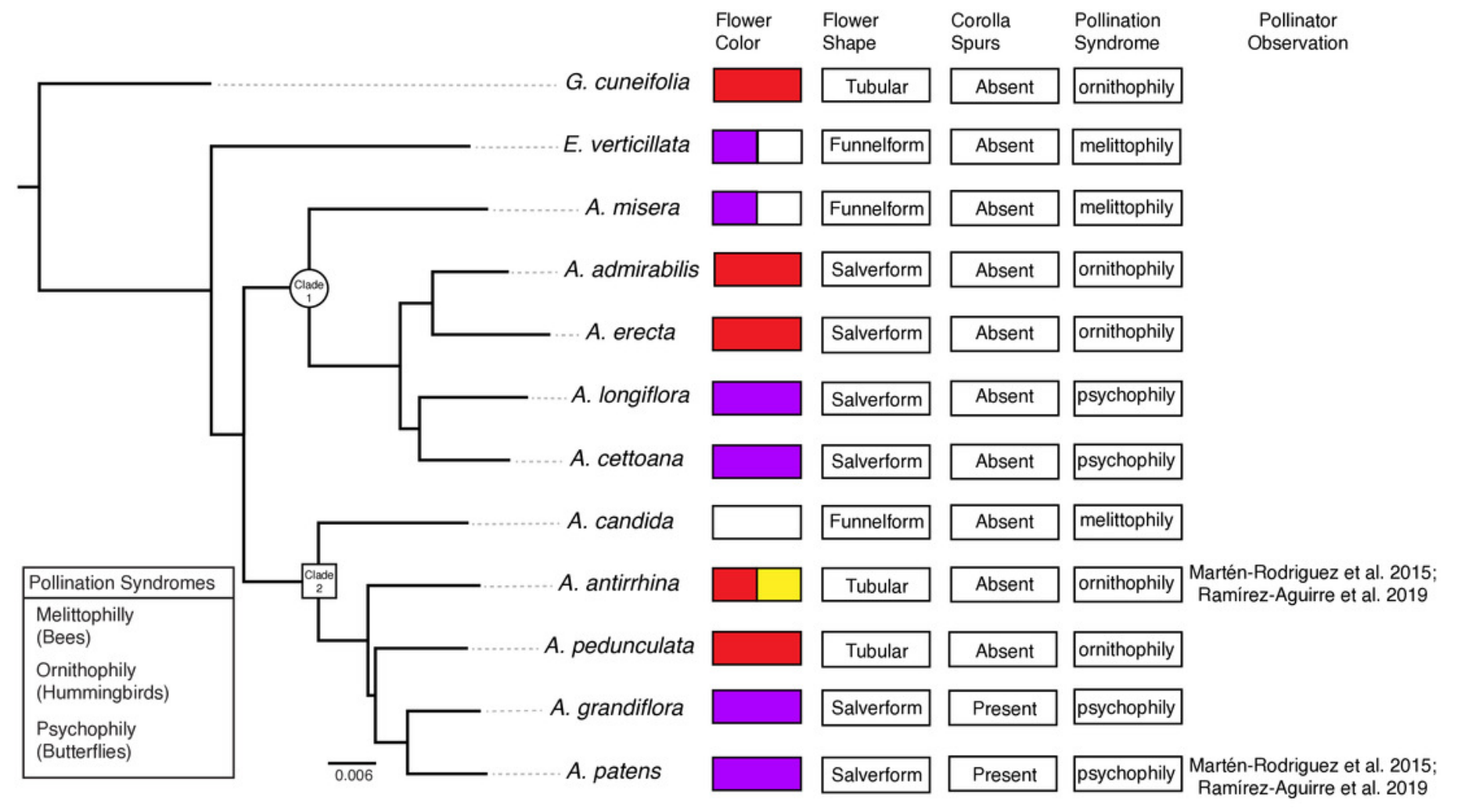


Figure 3

Co-expression clustering of the Bud and D stages

Hierarchical clustering by WGCNA showing co-expressed modules for $(\mathbf{A})$ the Bud stage and (B) the D stage. Each of the 9503 orthogroups were assigned to 65 and 62 modules, respectively, and the color rows underneath show the module assignment. 
A

\section{Cluster Dendrogram}

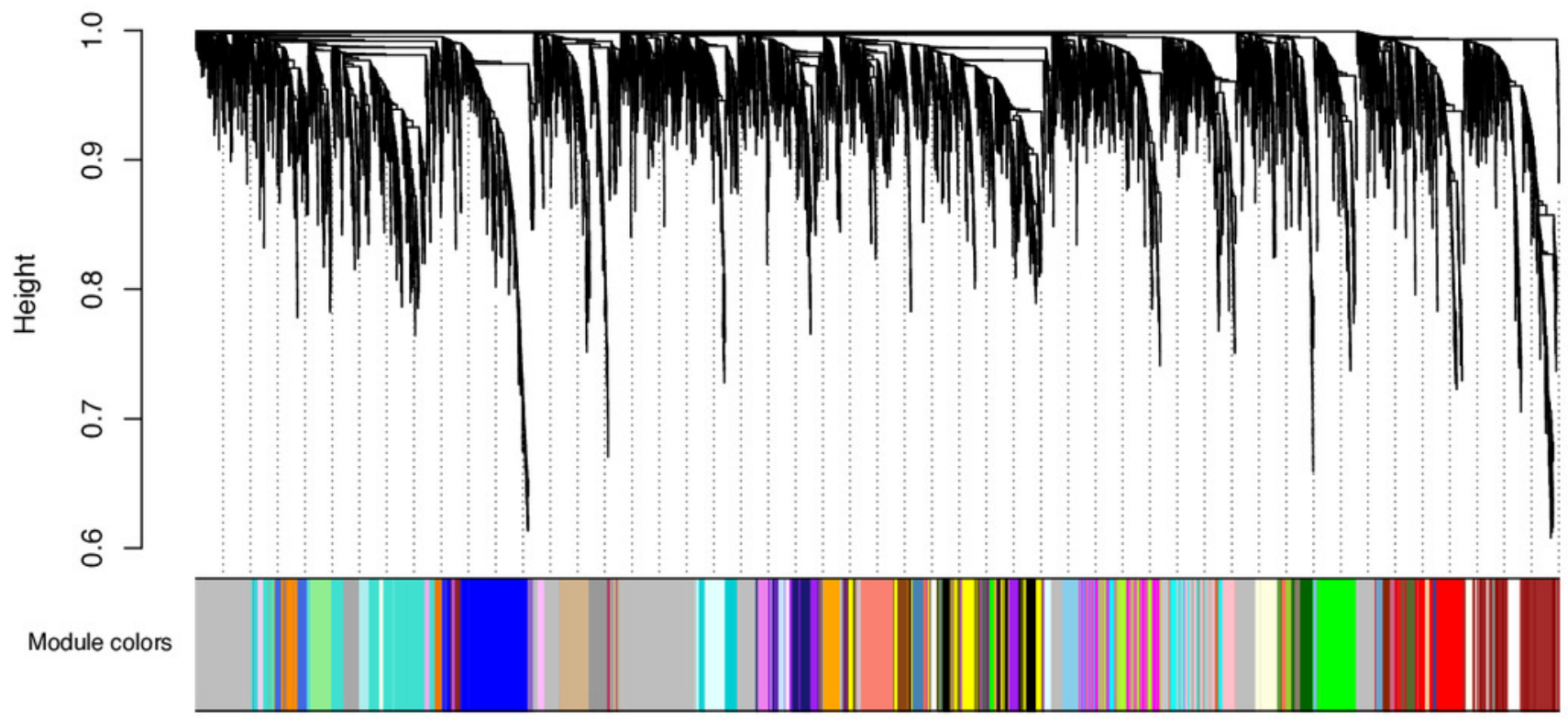

B

Cluster Dendrogram

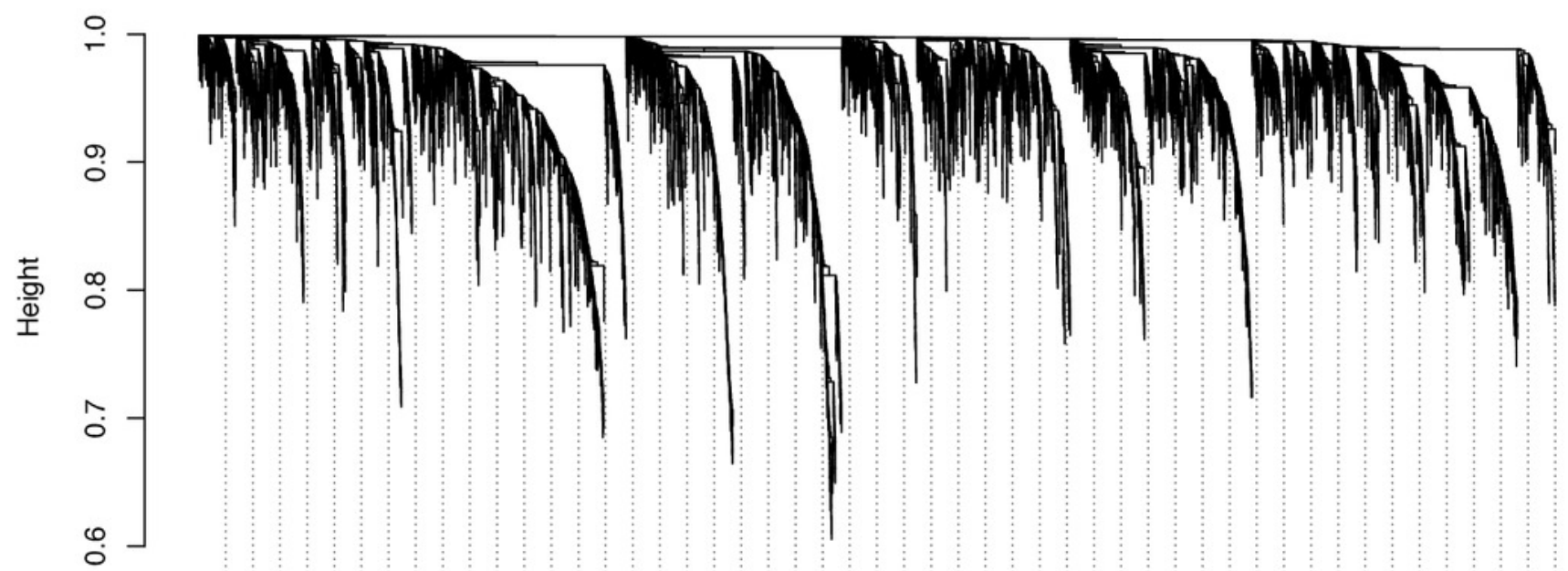

Module colors

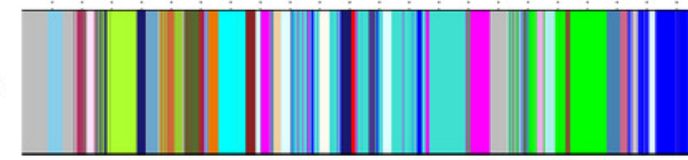




\section{Figure 4}

\section{Correspondence of the Bud and D stage networks}

Each row corresponds to one Bud stage module (labeled by module name and module size), and each column corresponds to one $\mathrm{D}$ stage module (labeled by module name and module size). Numbers in the table indicate gene counts in the intersection of the corresponding modules. Coloring of the table encodes $-\log (p)$, with $p$ being the Fisher's exact test $p$-value for the overlap of the two modules. The stronger the red color, the more significant the overlap.

\section{Correspondence of Bud and D stage modules}

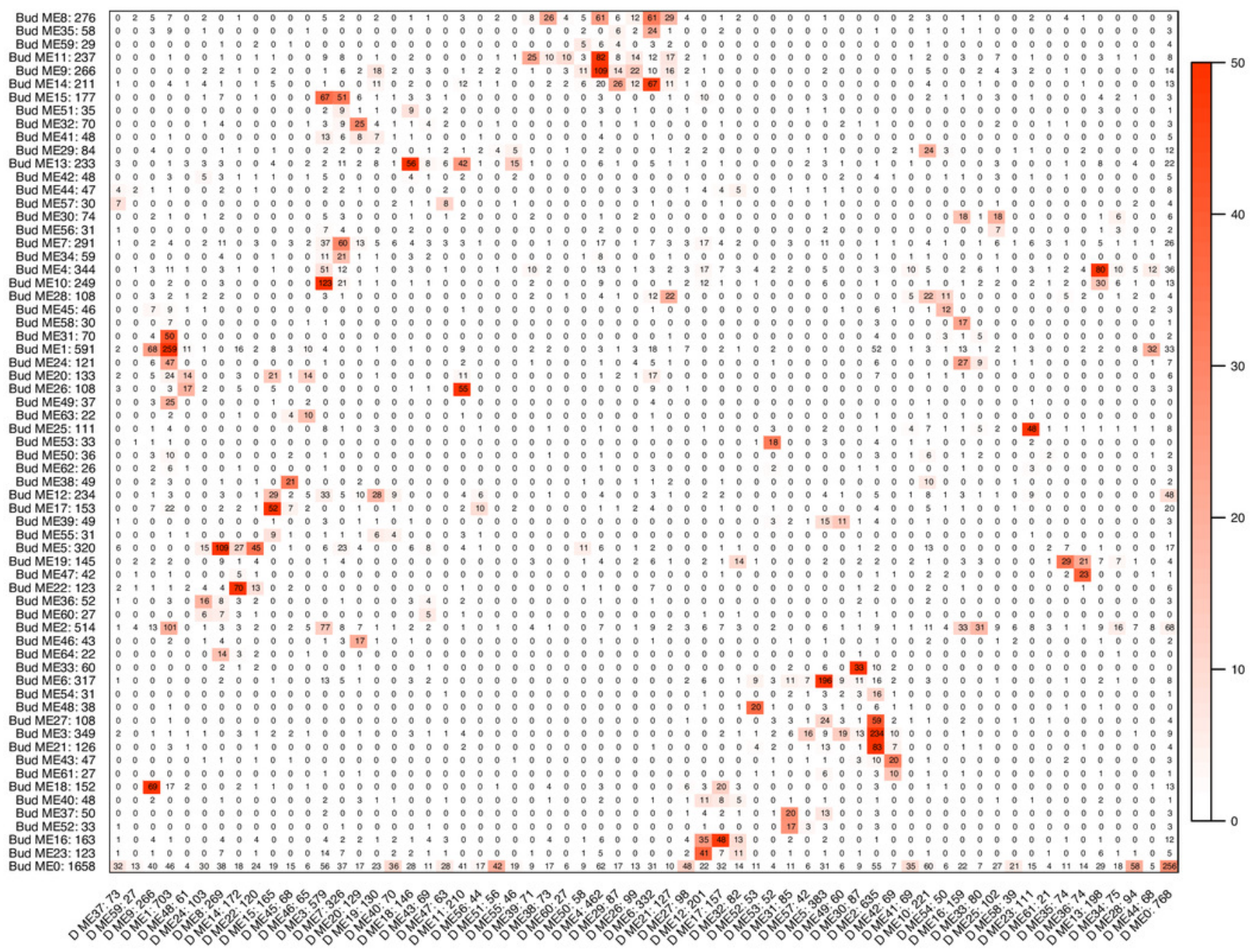




\section{Figure 5}

Summary of Bud stage network module association to floral traits and pollination syndrome

Each row represents an individual module in the Bud stage network and shows the total size (column All), the number of hubs (column Hubs), and whether the module is preserved in the D stage. Each column in the central table represents the floral traits (Pollination Syndrome, Flower color, Flower shape, and Spurs) that were tested for an association to module eigengenes using MCMCgImm. The eigengene expression is indicated for each module in which all three replicates from an individual species had $>1.0$ or $<-1.0$ expression. An association was considered strong when both MCMCgImm results were significant and the eigengene expression was $>1.0$ or $<-1.0$ in multiple species that shared the significant trait. An association was considered weak when MCMCgImm results were significant but the eigengene expression was $>1.0$ or $<-1.0$ in a single species or no species that shared the significant trait. Eigengene expression for species that share traits to those found significant by MCMCglmm are in bold. 


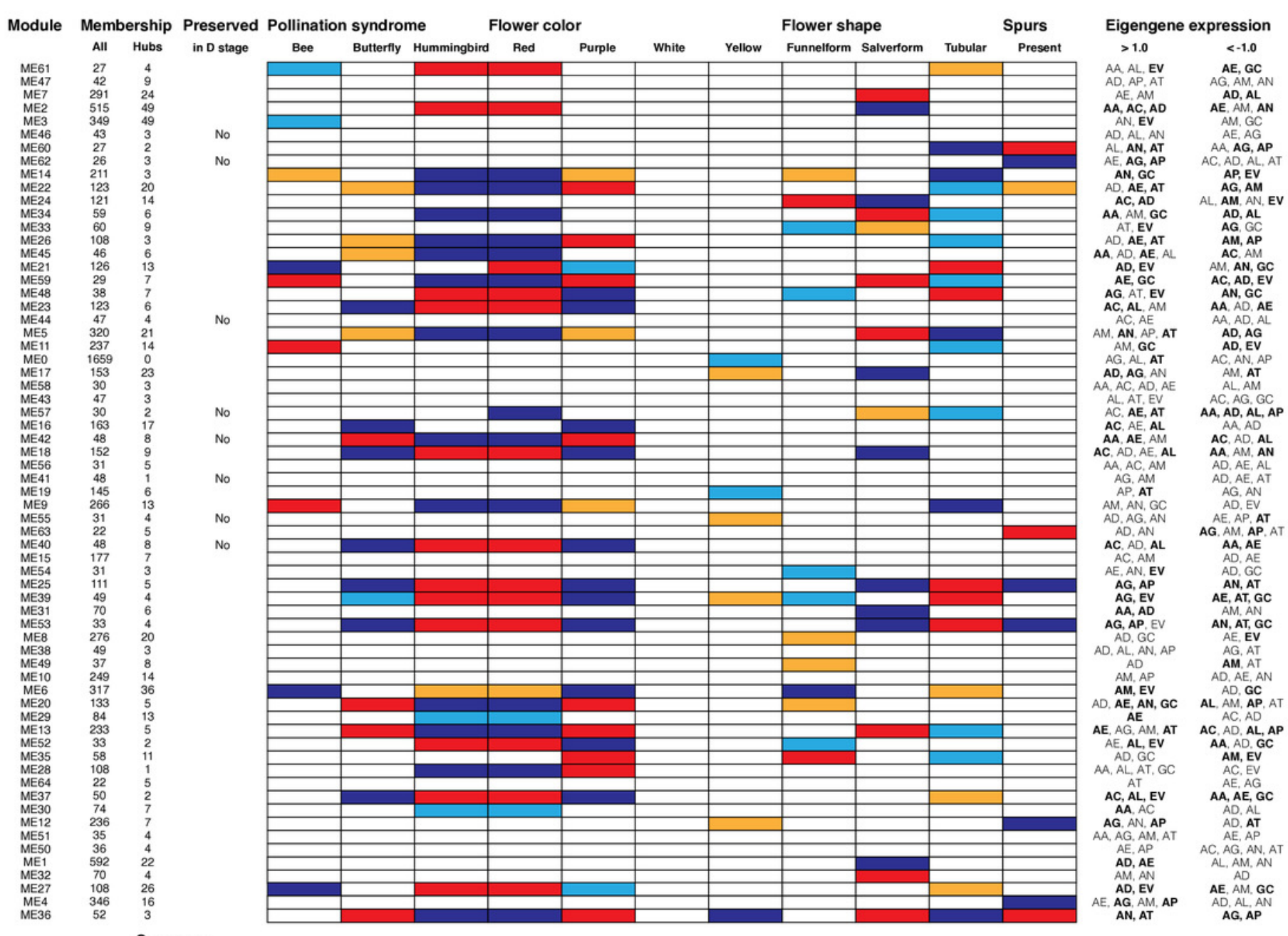

\section{Summary} \begin{tabular}{rl|c|c|c|c|c|c|c|c|c|c|c|} 
Number modules correlated & $3(4.6 \%)$ & $7(10.8 \%)$ & $13(20 \%)$ & $14(21.5 \%)$ & $11(16.9 \%)$ & 0 & $1(1.5 \%)$ & $1(1.5 \%)$ & $8(12.3 \%)$ & $5(7.7 \%)$ & $5(7.7 \%)$ \\
\cline { 2 - 9 } Number orthogroups correlated & $551(5.8 \%)$ & $680(7.2 \%)$ & $1736(18.3 \%)$ & $1766(18.6 \%)$ & $1117(11.8 \%)$ & 0 & $52(0.06 \%)$ & $317(3.3 \%)$ & $1747(18.4 \%)$ & $876(9.2 \%)$ & $752(7.9 \%)$ \\
\cline { 2 - 8 }
\end{tabular}

\section{Strongly positive $\square$ Weakly positive $\square$ Strongly negative $\square$ Weakly negative}




\section{Figure 6}

Summary of $D$ stage network module association to floral traits and pollination syndrome

Each row represents an individual module in the $D$ stage network and shows the total size (column All), the number of hubs (column Hubs), and whether the module is preserved in the Bud stage. Each column in the central table represents the floral traits (Pollination Syndrome, Flower color, Flower shape, and Spurs) that were tested for an association to module eigengenes. The eigengene expression is indicated for each module in which all three replicates from an individual species had $>1.0$ or $<-1.0$ expression. An association was considered strong when both MCMCgImm results were significant and the eigengene expression was $>1.0$ or $<-1.0$ in multiple species that shared the significant trait. An association was considered weak when MCMCgImm results were significant but the eigengene expression was $>1.0$ or $<-1.0$ in a single species or no species that shared the significant trait. Eigengene expression for species that share traits to those found significant by MCMCgImm are in bold. 


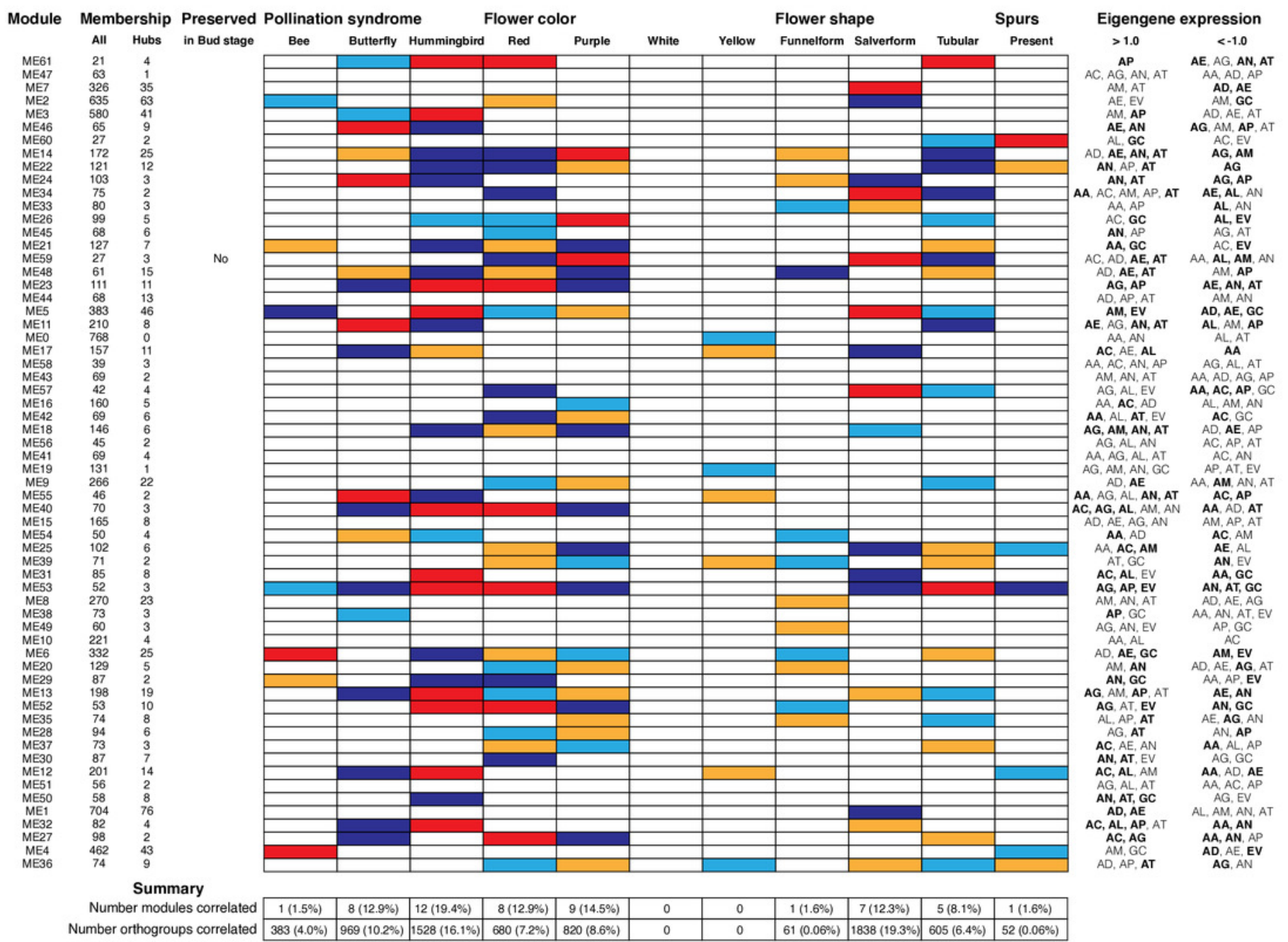

\section{Strongly positive $\square$ Weakly positive $\square$ Strongly negative $\square$ Weakly negative}

Abbreviations: AA, A. admirabilis; AC, A. cettoana; AD, A. candida; AE, A. erecta; AG, A. grandiflora; AL, A. longiflora; AM, A. misera, AN, A. pedunculata; AP, A. patens; AT, A. antirrhina; EV, E. verticillata; GC, G. cuneifolia. 


\section{Figure 7}

Relationship between orthogroup dN/dS (omega) and orthogroup connectivity

(A) Bud stage network. (B) D stage network. Each point represents an individual orthogroup.

The red line indicates the linear regression line for the relationship between $\mathrm{dN} / \mathrm{dS}$ and connectivity. The dotted line indicates $\mathrm{dN} / \mathrm{dS}=1$ with orthogroups above this line considered to be under relaxed or positive selection.

A

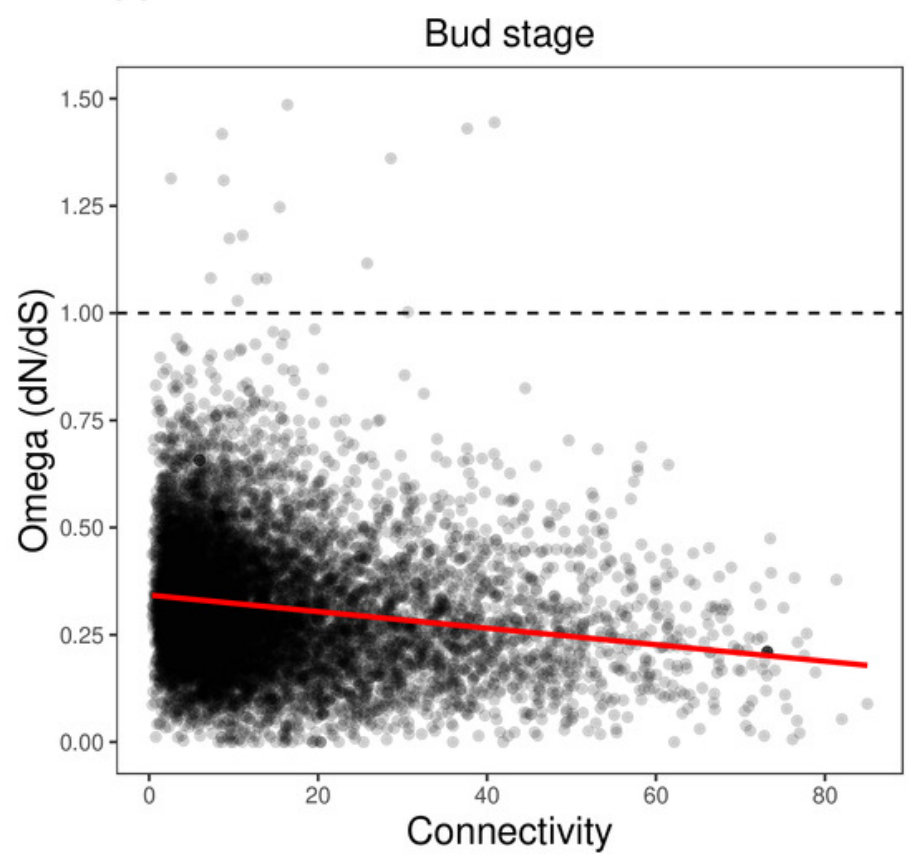

B

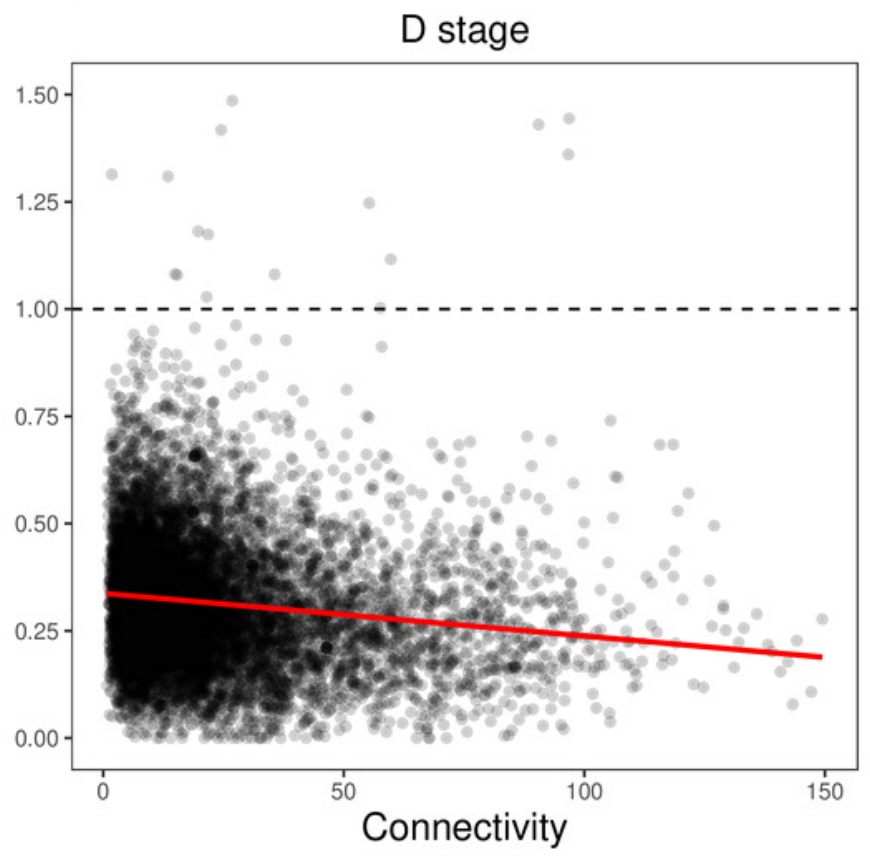

